\title{
Supercurrent decay in ballistic magnetic Josephson junctions
}

\author{
Hervé Ness ${ }^{1 凶}$, Ivan A. Sadovskyy $\mathbb{D}^{2 凶}$, Andrey E. Antipov ${ }^{2 凶}$, Mark van Schilfgaarde ${ }^{1,3 凶}$ and Roman M. Lutchyn ${ }^{2 凶}$
}

We investigate transport properties of ballistic magnetic Josephson junctions and establish that suppression of supercurrent is an intrinsic property of the junctions, even in absence of disorder. By studying the role of ferromagnet thickness, magnetization, and crystal orientation we show how the supercurrent decays exponentially with thickness and identify two mechanisms responsible for the effect: (i) large exchange splitting may gap out minority or majority carriers leading to the suppression of Andreev reflection in the junction, (ii) loss of synchronization between different modes due to the significant dispersion of the quasiparticle velocity with the transverse momentum. Our results for $\mathrm{Nb} / \mathrm{Ni} / \mathrm{Nb}$ junctions are in good agreement with recent experimental studies. Our approach combines density functional theory and the Bogoliubov-de Gennes model and opens a path for material composition optimization in magnetic Josephson junctions and superconducting magnetic spin valves.

npj Computational Materials (2022)8:23; https://doi.org/10.1038/s41524-021-00694-3

\section{INTRODUCTION}

Coherent quantum tunneling of Cooper pairs through a thin barrier is one of the first examples of macroscopic quantum coherent phenomena. As predicted by Josephson more than 50 years ago $^{1}$, it has important applications in quantum circuits used in metrology, quantum sensing, and quantum information processing $^{2}$.

Most of the previous studies focused on conventional Josephson junctions (JJs) consisting of two s-wave superconductors $(S)$ that are connected by an insulating (I) or a normal $(\mathrm{N})$ region $^{3,4}$. The flow of supercurrent through a JJ depends on the superconducting phase difference $\phi$ between two superconductors and, in general, is characterized by the current-phase relationship $J(\phi)$ (CPR). In conventional JJs CPR should be periodic with $2 \pi, I(\phi)=I(\phi+2 \pi)$ which follows from the Bardeen-Cooper-Schrieffer theory ${ }^{5}$. This result is a manifestation of a $2 e$ charge of Cooper pairs and is used in metrology to measure electron charge. Time-reversal symmetry requires that $I(\phi)=-I(-\phi)$ which imposes a constraint that the supercurrent should be zero for $\phi=\pi n$ where $n$ is an integer. In general, CPR can be expanded in Fourier harmonics, $I(\phi)=\sum_{n} I_{n} \sin (n \phi)$

In many cases, however, CPR is well approximated by the first harmonic $I(\phi) \approx I_{\mathrm{c}} \sin (\phi)$ with $I_{\mathrm{c}}$ being the maximum supercurrent that can flow through the junction, i.e., the critical current. At a microscopic level, the supercurrent through a short SNS junction is determined by bound states forming in the constriction due to Andreev's reflection at the NS interfaces. In the Andreev reflection process, an incident electron-like quasiparticle with spin $\uparrow$ gets reflected at the NS interface as a hole-like quasiparticle with spin $\downarrow$ and a Cooper pair is emitted into the condensate. When timereversal symmetry is not broken, electrons and holes propagate with the same velocity in the normal region. In this case, no phase shift accumulates between this pair of quasiparticles along their trajectories in the $\mathrm{N}$ region, and the sign of $I_{\mathrm{c}}$ is fixed. When $I_{\mathrm{C}}>0$ we refer to this case as 0 -junction.

In a magnetic Josephson junction (MJJ), exchange splitting breaks time-reversal symmetry and leads to an interesting interplay of superconductivity and magnetism ${ }^{4,6-9}$. In superconductor-ferromagnet-superconductor (SFS) junctions [Fig. 1a] the correlated quasi-particles and quasi-holes forming Andreev bound states propagate through the junction under the exchange field of the ferromagnet (F). In many ferromagnets, such as $\mathrm{Fe}$ or $\mathrm{Ni}$, the exchange splitting is large (of the order of eV) and significantly perturbs the band structure of metal and, consequently, significantly modifies Fermi velocities of minority (spin $\downarrow$ ) and majority (spin $\uparrow$ ) carriers. Strong time-reversal symmetry breaking leads to the appearance of characteristic superconducting correlations with oscillatory dependence determined by the difference in wavenumbers, $k_{\mathrm{F}}^{\uparrow}-k_{\mathrm{F}}^{\downarrow 10,11}$. This effect opens a possibility for the supercurrent reversal as a function of the thickness of the ferromagnetic region, the so-called Josephson $\pi$ junction ${ }^{12,13}$. The correlation between the phase shift of the supercurrent and the magnetization provides a possibility for realizing magnetic spin valves, see Fig. $1 \mathrm{~b}$, which may have promising novel applications for cryogenic superconducting digital technologies ${ }^{14-16}$. Understanding the microscopic physics of MJJs is of great scientific interest as well as technological importance. $0-\pi$ transitions in SFS junctions have been extensively studied experimentally since the early 2000s and have been observed in different material systems ${ }^{13,17-25}$. While qualitatively these observations are consistent with the previous phenomenological theories ${ }^{26-42}$ the roles of the microscopic band structure arising from the atomic lattice on the supercurrent suppression with junction thickness remain unclear. Our primary goal is to address these essential points. The supercurrent suppression that is exponential in the junction length is often associated with the presence of disorder in the ferromagnetic region ${ }^{6,43}$. However, significant supercurrent suppression can also appear in relatively clean metals (e.g., Ni) whose mean free path is larger than the junction thickness ${ }^{22,23,44}$.

Here, we study the suppression of the critical current in the MJJs shown in Fig. 1a and identify two microscopic mechanisms for its suppression, both a consequence of the band structure asymmetry of the majority and minority carriers in the $\mathrm{F}$ region. First, there is an asymmetry in the structure of the Fermi surface, see Fig. 2a. For certain bands and momenta, the Fermi surface present

\footnotetext{
${ }^{1}$ Department of Physics, King's College London, Strand Campus, London WC2R 2LS, UK. ${ }^{2}$ Microsoft Quantum, Microsoft Station Q, University of California, Santa Barbara, CA 93106, USA. ${ }^{3}$ National Renewable Energy Laboratory, Golden, CO 80401, USA. ${ }^{凶}$ email: herve.ness@kcl.ac.uk; ivan.sadovsky@gmail.com; andrey.antipov@microsoft.com; mark.vanmchilfgaarde@nrel.gov; roman.lutchyn@microsoft.com
} 


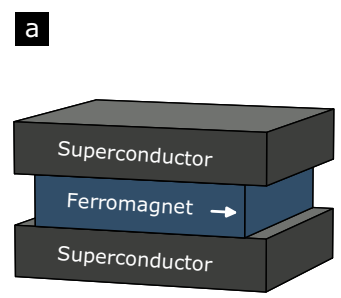

b

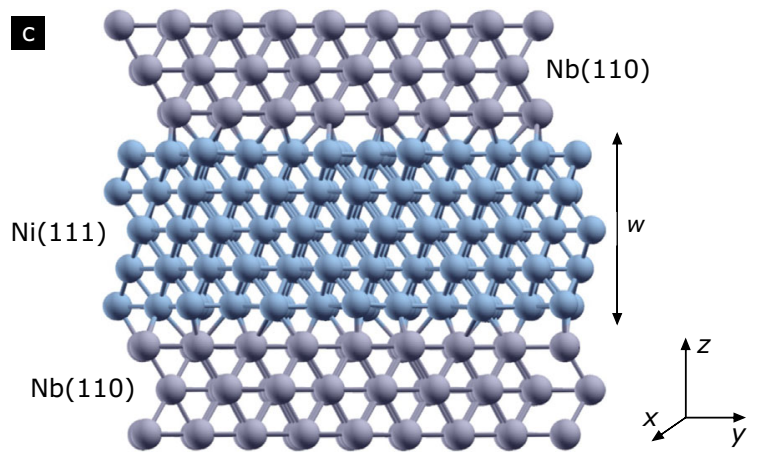

Fig. 1 MJJ stacks. a Schematic view of the SFS junction. b SFNFS junction. Arrows indicate possible magnetization of ferromagnets. The supercurrent through the spin-valve JJ depends on the relative magnetization of the ferromagnets, which governs the properties of the Josephson magnetic random-access memory (JMRAM) ${ }^{16}$. c Balland-stick representation of the $\mathrm{Nb}(110) / \mathrm{Ni}(111) / \mathrm{Nb}(110)$ junction with five layers of $\mathrm{Ni}$. $\mathrm{Nb}$ atoms are light grey, $\mathrm{Ni}$ atoms are blue. The top and bottom atomic planes of $\mathrm{Nb}(110)$ are repeated periodically in the $z$-direction to create the semi-infinite $\mathrm{Nb}$ leads of the junction through which the current flows. Periodic boundary conditions are used in the $x y$-plane. The corresponding reciprocal space defines two-dimensional $\mathbf{k}_{\|}=\left(k_{x}, k_{y}\right)$ vectors, i.e., the transverse modes, used in the calculations.

in one spin channel may be absent in the other. This is typical in ferromagnetic materials like $\mathrm{Fe}, \mathrm{Co}$, and $\mathrm{Ni}$ because the bandwidth for $d$-electrons is relatively small and is often comparable to the exchange splitting. As a result, the wavenumber of one of the constituent quasi-particles forming Andreev bound states in MJJ becomes imaginary and the supercurrent becomes suppressed. We label this scenario as mechanism (i). In a second mechanism (ii), we show there is a dephasing of a harmonic signal originating from different Fourier components of the supercurrent due to the Fermi velocity dispersion, see Fig. 3a. Both these mechanisms lead to an exponential suppression of the supercurrent, which was previously believed to occur due to the presence of disorder in the magnetic region. We show that band structure effects are important and maybe even dominant in many cases.

In order to capture a realistic band structure, we develop a microscopic theory for the supercurrent in realistic MJJs. We use a combination of density functional theory (DFT) and Bogoliubov-de Gennes (BdG) model to investigate the $0-\pi$ transition in realistic material stacks of $\mathrm{Nb} / \mathrm{Ni} / \mathrm{Nb}$ junctions in the clean limit. This method allows one to predict and explain key properties of MJJs such as the period and decay of the critical current oscillations with the ferromagnet thickness.

\section{RESULTS}

\section{Qualitative discussion and main results}

In this section, we describe basic concepts for the supercurrent flow in MJJs and summarize our results. Our main qualitative conclusions are supported by microscopic calculations for $\mathrm{Nb} /$ $\mathrm{Ni} / \mathrm{Nb}$ MJJs. Ni appears to have a fairly long mean free path $I_{\text {MFP }} \approx 60 \AA$ (see estimations in Supplementary Note 1 ), which is a

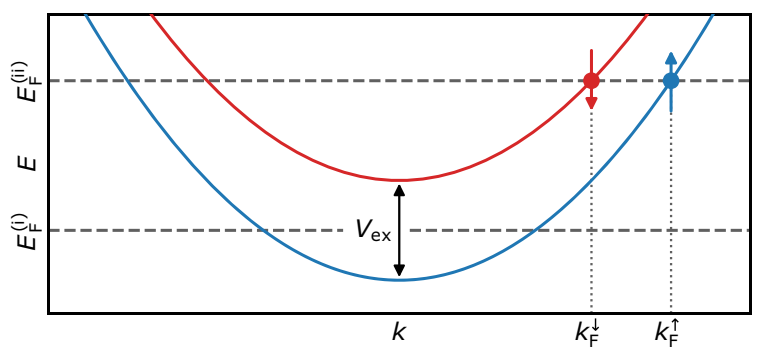

b

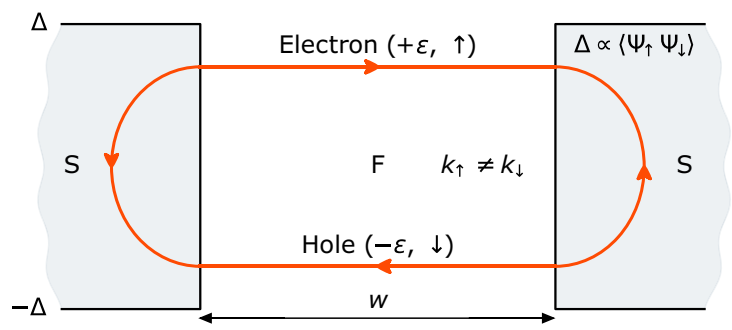

Fig. 2 Bound states in SFS junctions. a Simplified band structure of a ferromagnet, with the majority and minority bands, split by $V_{\mathrm{ex}} \cdot k_{\mathrm{F}}^{\uparrow}$ and $k_{F}^{\downarrow}$ are the Fermi momenta for the majority and minority carriers, respectively. Fermi level $E_{\mathrm{F}}^{(\mathrm{i})}$ corresponds to large $V_{\mathrm{ex}}$, where the minority band is pushed above $E_{\mathrm{F}}^{(\mathrm{i})}$. $E_{\mathrm{F}}^{(\mathrm{ii})}$ corresponds to small $V_{\mathrm{ex}}$. Thus, the propagation of minority quasiparticles is characterized by an imaginary momentum $k_{F}^{\downarrow}$ and is suppressed. b Supercurrent in SFS junction is carried by Andreev bound states localized in the junction. Solid red line represents a quasi-classical trajectory corresponding to an Andreev bound state. The spectrum of Andreev states depends on the relative superconducting phase difference across the junction as well as the phase, $\delta \varphi=\left|k_{\mathrm{F}}^{\uparrow}-k_{\mathrm{F}}^{\downarrow}\right| w$, accumulated due to the difference of Fermi momenta for the majority and minority carriers. Note that in scenario (i) the propagation of minority carriers is suppressed leading to an overall exponential decay of the supercurrent with $w$. This is to be contrasted with the normal transport through the junction.

comparable or larger than the typical thickness of the ferromagnet used in recent experiments ${ }^{22,23}$. Therefore, the motion of quasiparticles in $\mathrm{Nb} / \mathrm{Ni} / \mathrm{Nb}$ junction is quasi-ballistic, and our method is applicable to this system. Most of this paper focuses on clean $\mathrm{Nb} / \mathrm{Ni} / \mathrm{Nb}$ junctions.

First it is illuminating to consider a toy model, a onedimensional SFS junction, and calculate the supercurrent in such a system for different Fermi energies, see Fig. 2a. Using the results of ref. ${ }^{45}$, one finds that both majority and minority spin bands are both occupied in the limit $V_{\mathrm{ex}} / E_{\mathrm{F}} \ll 1$ [i.e., scenario (ii) in Fig. 2a] the supercurrent does not decay with ferromagnet thickness at zero temperature,

$$
I(\phi)=\frac{2 e \Delta}{\hbar}\left\{\begin{array}{cc}
\cos \delta \varphi \sin \frac{\phi}{2}, & 0<\phi<\pi-2 \delta \varphi, \\
-\sin \delta \varphi \cos \frac{\phi}{2}, & \pi-2 \delta \varphi<\phi<\pi+2 \delta \varphi, \\
-\cos \delta \varphi \sin \frac{\phi}{2}, & \pi+2 \delta \varphi<\phi<2 \pi .
\end{array}\right.
$$

Here perfect interface transparency $\mathcal{T}$ is assumed, $\mathcal{T} \approx 1$. The phase offset $\delta \varphi$ originates from the Fermi momentum difference of a quasi-particle and a quasi-hole forming Andreev bound state in the junction, see Fig. $2 \mathrm{~b}$, and is given by $\delta \varphi=\left|k_{\mathrm{F}}^{\uparrow}-k_{\mathrm{F}}^{\downarrow}\right| w \approx V_{\mathrm{ex}} w / \hbar v_{\mathrm{F}}$. The 0 - and $\pi$-junction regimes can be clearly identified at $\delta \varphi=0$ and $\delta \varphi=\pi / 2$, respectively. At the intermediate values $0<\delta \varphi<\pi / 2$, the CPR is anharmonic which is a generic feature at $0-\pi$ transition as shown below. In the low transparency regime, $\mathcal{T} \ll 1$, one would expect qualitatively similar results with the maximal critical current being suppressed $I_{\mathrm{c}} \sim(e \Delta / \hbar) \mathcal{T}$ but still independent of the ferromagnet thickness, $w$. 

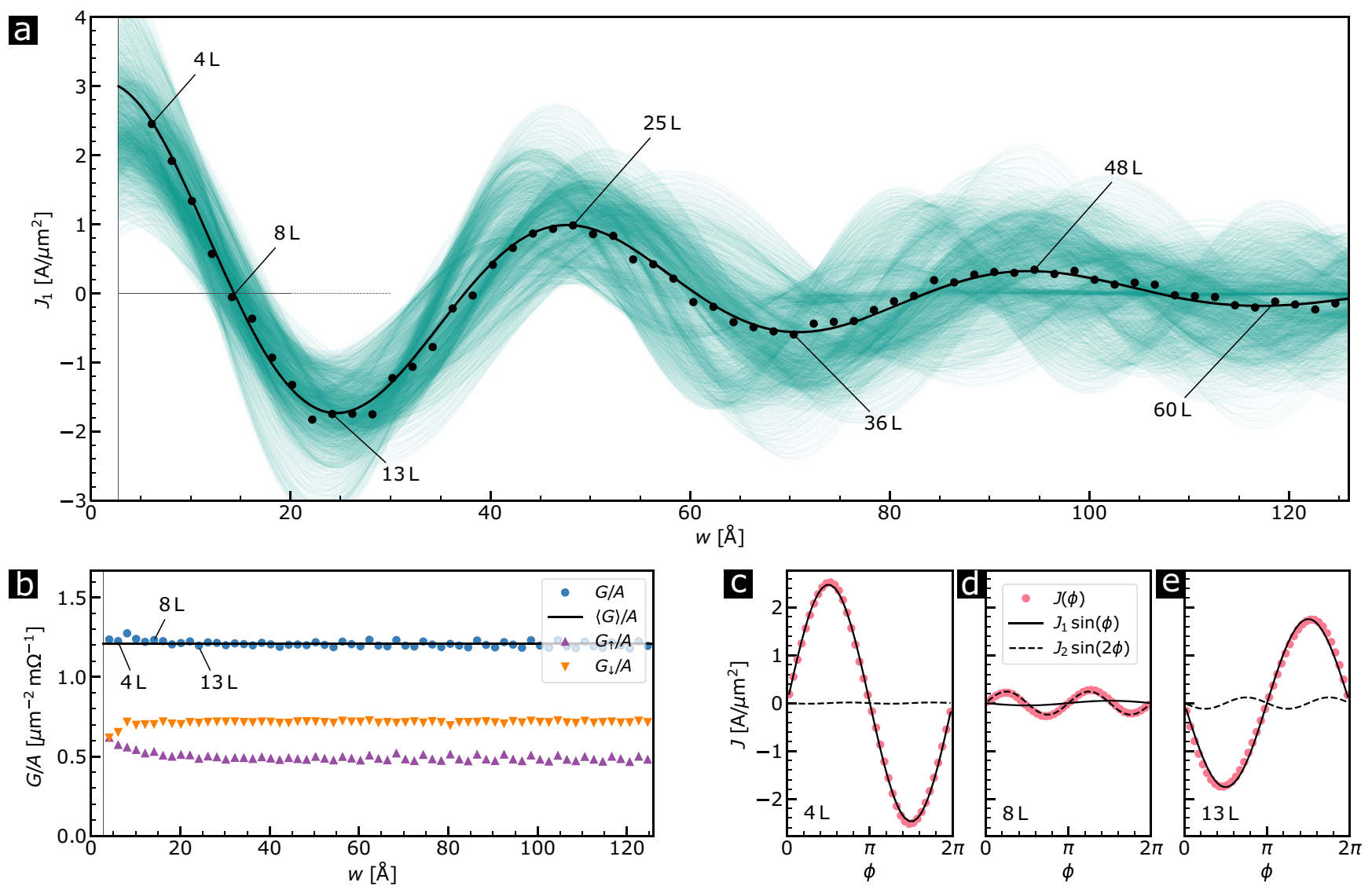

Fig. 3 Normal and superconducting current of $\mathbf{N b} / \mathbf{N i} / \mathbf{N b}$ junction. a First Fourier harmonic $J_{1}$ of the supercurrent density [Eq. (3), black circles] and it is fit [Eq. (6), solid black line] as a function of Ni layer thickness, $w$, calculated for the $\mathrm{Nb}(110) / \mathrm{Ni}(111) / \mathrm{Nb}(110)$ junctions shown in Fig. 1c. Green semitransparent curves correspond to $j_{1}^{\text {fit }}\left(\mathbf{k}_{\|}\right)$for individual $\mathbf{k}_{\| \cdot} \cdot \mathbf{b}$ Normal state conductance per unit of area as a function of $w$ for the majority $\left(G_{\uparrow}\right)$ and minority $\left(G_{\downarrow}\right)$ spins [Eq. (13)] as well as $G=G_{\uparrow}+G_{\downarrow}$. $G$ does not depend on $w$ and is approximated by the single value $\langle G\rangle$. c-e Supercurrent as a function of phase difference $\phi$ for $\mathbf{c} 4$ atomic layers of Ni (strong 0-junction regime), $\mathbf{d} 8$ layers (intermediate regime), and e 13 layers (strong $\pi$-junction regime). In the 0 - and $\pi$-junction regimes, the $J_{1}$ component dominates. In the intermediate regime, higherorder terms may prevail.

In the case of large exchange splitting $V_{\mathrm{ex}} / E_{\mathrm{F}} \gg 1$ [scenario (i) in Fig. 2a], the minority band may become unoccupied. Given that minority carriers are gapped out and their propagation through the junction is suppressed, the supercurrent decays exponentially with $w^{45}$

$I(\phi) \approx \frac{2 e \Delta}{\hbar} \exp (-\kappa w)\left[1-\frac{E_{\mathrm{F}}}{8 V_{\mathrm{ex}}} \sin ^{2}(k w)\right] \sin \phi$.

Here, $k=\sqrt{2 m^{*}\left(V_{\mathrm{ex}}-E_{\mathrm{F}}\right)} / \hbar$ and $k=\sqrt{2 m^{*}\left(V_{\mathrm{ex}}+E_{\mathrm{F}}\right)} / \hbar$ with $m^{*}$ being effective electron mass. One may notice the drastic difference between normal-state and superconducting transport in this case-the former is weakly affected (because majority and minority contributions are additive) whereas the supercurrent is strongly suppressed. In this case, the measurement of normalstate junction resistance does not necessarily predict the magnitude of the supercurrent through the junction.

We now generalize above results for the realistic threedimensional (3D) geometry and material composition of the MJJ. In the clean limit, the supercurrent $I(\phi)$ in the short-junction limit ( $w$ much smaller than the coherence length of the superconductor) is obtained from the spectrum of the Andreev bound states $\varepsilon_{v}\left(\phi, \mathbf{k}_{\|}\right)$localized in the junction ${ }^{3}$ which now also depends on the parallel momentum $\mathbf{k}_{\|}$. The supercurrent density $J(\phi)$ per junction area $A$ is $J(\phi)=I(\phi) / A$. For the junction with periodic atomic structure in $x y$-plane the supercurrent density at zero temperature is given by

$$
J(\phi)=-\frac{e}{\hbar} \int_{\mathrm{BZ}} \frac{d \mathbf{k}_{\|}}{(2 \pi)^{2}} \sum_{v>0} \frac{\partial \varepsilon_{v}\left(\phi, \mathbf{k}_{\|}\right)}{\partial \phi},
$$

where the $\mathbf{k}_{\|}$integration is performed over the Brillouin zone (BZ) of the corresponding surface supercell of area, $A$, and the sum is carried over positive quasiparticle energies, $\varepsilon_{v}\left(\phi, \mathbf{k}_{\|}\right)>0$. Note that we use spin-resolved $\varepsilon_{v}$ and therefore omit spin prefactor 2 in Eq. (3). The derivative is periodic in $\phi$ and can be represented as a Fourier series

$$
-\frac{e}{\hbar} \frac{\partial \varepsilon_{v}\left(\phi, \mathbf{k}_{\|}\right)}{\partial \phi}=\sum_{n \geqslant 1} I_{n v}\left(\mathbf{k}_{\|}\right) \sin \left[n \phi+\delta \varphi_{n v}\left(\mathbf{k}_{\|}\right)\right],
$$

so that Eq. (3) can be written as

$$
J(\phi)=\int_{\mathrm{BZ}} \frac{d \mathbf{k}_{\|}}{(2 \pi)^{2}} \sum_{v>0} \sum_{n \geqslant 1} I_{n v} \sin \left[n \phi+\delta \varphi_{n v}\left(\mathbf{k}_{\|}\right)\right] .
$$

As we will show below, away from $0-\pi$ transition the supercurrent is dominated by the first $(n=1)$ harmonic. Therefore, we focus henceforth on the first harmonic contribution and drop $n$ index in the following discussion. Next, one may notice that the supercurrent amplitudes $I_{v}\left(\mathbf{k}_{\|}\right)$and phase offsets $\delta \varphi_{v}\left(\mathbf{k}_{\|}\right)$depend on the parallel momentum $\mathbf{k}_{\|}$. One may include this dependence and define an effective Fermi energy $E_{F}\left(\mathbf{k}_{\|}\right)$that counts the energy corresponding to $\mathbf{k}_{\|}$in each band of the ferromagnet from the bottom of the band. Depending on $E_{\mathrm{F}}\left(\mathbf{k}_{\|}\right)$and $V_{\mathrm{ex}}$ either scenario (i) or (ii) of Fig. 2a may be realized. 
H. Ness et al.

Table 1. Minimum, maximum, average, and root mean square of the Fermi velocities $v_{\mathrm{F}}$ for the majority and minority Fermi surfaces in bulk Ni.

\begin{tabular}{|c|c|c|c|c|c|c|c|c|c|c|c|c|c|}
\hline \multicolumn{9}{|c|}{ Majority $(\uparrow)$} & \multicolumn{5}{|c|}{ Minority $(\downarrow)$} \\
\hline 2 & & & & & & -1.59 & 0.68 & 12 & & & & & \\
\hline 3 & & & & & & -0.22 & 3.90 & 13 & 2.7 & 3.6 & 3.3 & 3.6 & 0.004 \\
\hline 4 & & & & & & -0.11 & 0.61 & 47 & 0.8 & 1.8 & 1.1 & 1.2 & 0.015 \\
\hline
\end{tabular}

$\rho\left(E_{\mathrm{F}}\right)$ is the density of states at the Fermi level, $\Delta E$ is the energy of the valence band maximum relative to $E_{\mathrm{F}}$ for the majority bands not crossing $E_{\mathrm{F}}, m^{*}\left(m_{e}\right)$ is the effective (bare) electron mass, and $\kappa$ estimates the decay exponent of the evanescent mode for a given band at $E_{\mathrm{F}}$.

Thus, it is important to compare the exchange splitting with the bandwidth of $d$-character states in transition metals to make sure that a perturbation theory in $V_{\text {ex }}$ is justified. Assuming it is the case, one can estimate the phase offset $\delta \varphi_{\nu}\left(\mathbf{k}_{\|}\right)$by expanding in exchange splitting to find

$$
\delta \varphi_{v}\left(\mathbf{k}_{\|}\right) \approx v_{\mathrm{ex}} w / \hbar v_{z v}\left(\mathbf{k}_{\|}\right) .
$$

In general, the dependence of $v_{z v}$ on $\mathbf{k}_{\|}$is complicated, especially in spd-transition metals. The combination of complicated amplitude and phase offset dependence on $\mathbf{k}_{\|}$leads to a non-trivial supercurrent dependence on the ferromagnet thickness $w$. As shown in Fig. 3a, the critical current decays with $w$ for the $\mathrm{Nb}(110) /$ $\mathrm{Ni}(111) / \mathrm{Nb}(110)$ junctions. We analyze the details of the decay and perform an exponential fit in Section "Details of the supercurrent". At small thicknesses, below $30 \AA$, this decay originates from the evanescent modes corresponding to gapped out minority or majority carriers which cannot propagate through the junction, see Table 1. This is the mechanism (i) discussed above. At larger thicknesses (i.e., $w \gtrsim 50 \AA$ ) a loss of synchronization between different modes due to the dispersion of $v_{z v}\left(\mathbf{k}_{\|}\right)$becomes important. This second mechanism (ii) has been previously discussed in the literature ${ }^{6,12}$ under assumptions of a single spherical Fermi surface and a small uniform exchange splitting $V_{\text {ex }}$ in the magnetic region. Within these assumptions, one finds that critical current should decay algebraically with the thickness $w$ of a magnetic layer ${ }^{12}$. However, as we show below most of these assumptions do not apply to realistic SFS junctions involving transition metals. Thus, in order to understand CPR in realistic MJJs, one needs to use accurate ab initio methods, which capture the physical effects described above.

To make a connection between the decay seen in Fig. 3 and the mechanisms responsible for it, Fig. 4a presents the energy band structure of bulk Ni. It is probably the highest fidelity band structure available: it very closely reproduces ARPES data in both majority and minority spin bands, with exchange splitting $V_{\mathrm{ex}}=$ $0.3 \mathrm{eV}^{46}$, and should be an excellent predictor of the real Fermi surface and velocities in $\mathrm{Ni}$.

Here, we use this potential to analyze the bulk Ni Fermi surface [Fig. 4b] and Fermi velocities (Table 1). Counting from the bottom $s$-band, bands of Ni $d$ character are bands 2 to 6 . These bands are nearly full: only majority band $\sigma^{\uparrow}$ and minority bands $3^{\downarrow}-6^{\downarrow}$ cross the Fermi level $E_{\mathrm{F}}$. Only band 6 has both majority and minority carriers at the Fermi surface. Bands $\sigma^{\uparrow}$ and $\sigma^{\downarrow}$ have roughly the same shape and the energy splitting is approximately constant and equal to $V_{\text {ex }}$ (see the right panel of Fig. 4 in ref. ${ }^{46}$ ). Beyond this, however, the correspondence between the Ni band structure and a simple parabolic band structure deviates substantially, in two ways that critically affect the analysis. First, the Fermi velocity, $v_{\mathrm{F}}=\left.\hbar^{-1}(\partial E / \partial k)\right|_{k=k_{F}}$, is not fixed even for a single pocket: it varies in band $\sigma^{\uparrow}$ by a factor of 2 [see Fig. 4 and Table 1]. Accordingly, the splitting $k_{\mathrm{F}}^{\uparrow}-k_{\mathrm{F}}^{\downarrow}$ between bands $\sigma^{\uparrow}$ and $\sigma^{\downarrow}$ varies by a factor of two
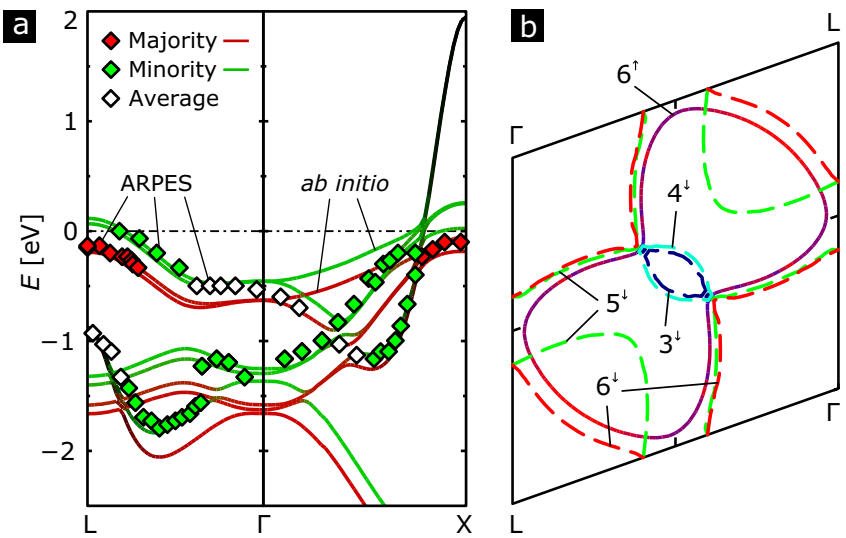

Fig. 4 Band structure. a Electronic band structure of bulk Ni (solid lines) calculated from first principles including many-body effects, see ref. ${ }^{46}$. It is the highest-fidelity available and is very close to ARPES data (diamonds) in both majority (red) and minority (green) spin bands, with exchange splitting $V_{\text {ex }}=0.3 \mathrm{eV}$. b Majority (solid line) and minority (dashed line) Fermi surfaces of bulk Ni in the $\mathbf{k}_{\|}$ plane (with $k_{z}=0$ ) corresponding to the (111) plane direction used for the stacking of the $\mathrm{Nb} / \mathrm{Ni} / \mathrm{Nb}$ junctions, as discussed in the text. Axes correspond to two $\Gamma$ - $L$ lines: the Fermi surface has a threefold symmetry in the entire plane. Majority band $6^{\uparrow}$ is depicted as a solid red-blue line with color interpolating between red and blue depending on the Fermi velocity $\hbar^{-1} \partial E / \partial k$, which ranges between $3 \times 10^{5} \mathrm{~m} / \mathrm{s}$ (red) and $6 \times 10^{5} \mathrm{~m} / \mathrm{s}$ (blue). Minority bands are shown by dashed lines: $3^{\downarrow}$ (blue), $4^{\downarrow}$ (cyan), $5^{\downarrow}$ (green), and $6^{\downarrow}$ (red).

as expected from the twofold variation in $v_{\mathrm{F}}$. Second, bands $3^{\downarrow}-5^{\downarrow}$ have no majority counterpart at $E_{\mathrm{F}}$ indicating that the wave number of bands $3^{\uparrow}-5^{\uparrow}$ is complex. This is the origin for the exponential decay in scenario (i) in Fig. 2a as noted above: a large portion of Andreev levels is carried by Cooper pairs made of single-particle wave functions with one or both of $k_{\mathrm{F}}^{\uparrow}$ and $k_{\mathrm{F}}^{\downarrow}$ having an imaginary component. The magnitude of $\operatorname{Im} k$ depends on the particular mode and $\mathbf{k}_{\|}$leading to a distribution of decay exponents. The slowest decay in each of these evanescent modes can be estimated from the distance $\Delta E$ of the closest approach to $E_{\mathrm{F}}$ and the effective mass $\mathrm{m}^{*} / m_{e}$, using $\hbar^{2} k_{\min }^{2} / 2 m^{*}=\Delta E$ and decay $\kappa=2 \pi / \operatorname{Im}\left(k_{\min }\right)$, see Table $1 .\left(\mathrm{m}^{*} / \mathrm{m}_{e}\right.$ is found to be highly anisotropic, so only the effective transport mass $m^{*}=$ $3\left[1 / m_{1}^{*}+1 / m_{2}^{*}+1 / m_{3}^{*}\right]^{-1}$ is shown.) $k$ is only a rough measure of the evanescent mode decay for a given band.

Let us now focus on the mechanism (ii) for the supercurrent decay, i.e., loss of synchronization between different transverse modes. This mechanism is well-known in diffusive systems where quasiparticle trajectory is random and thus the phase offset $\delta \varphi$ accumulated along such a trajectory also gets randomized. Thus, upon averaging Eq. (3) over different disorder realizations, 
one ends up with exponentially decaying critical current ${ }^{6}$. Previously, such a suppression of the supercurrent with junction thickness, $w$, was often associated with impurity scattering in the ferromagnet. Here, we demonstrate that this dephasing mechanism can also appear in clean systems (where quasiparticle trajectory is well defined) due to the dispersion of the velocity $v_{z v}$ with an in-plane momentum $\mathbf{k}_{\|}$. Specifically, we find that, in the $\mathrm{Nb} / \mathrm{Ni} / \mathrm{Nb}$ junctions, this mechanism becomes relevant for junctions thicker than $50 \AA$, see Fig. 3. In SFS junctions the combination of disorder in the ferromagnet, interface scattering as well band-structure-induced dephasing ultimately determines the magnitude of the supercurrent. However, we believe that band structure effects provide an upper bound on the magnitude of the critical current as a function of $w$.

Next, we present numerical results which support the qualitative discussion presented above.

\section{Choice of MJJ stack}

It is illuminating to compare our numerical simulations for the supercurrent with the experimental measurements involving quasi-ballistic MJJs. As previously discussed, we believe that the $\mathrm{Nb} / \mathrm{Ni} / \mathrm{Nb}$ junctions represent a good model system for which experimental data are readily available ${ }^{15,22,23}$. The bestperforming stacks consist of $\mathrm{Nb}(110) / \mathrm{Cu} / \mathrm{Ni}(111) / \mathrm{Cu} / \mathrm{Nb}(110)$. $\mathrm{Cu}$ spacer layers seem to be essential to get strong supercurrent, likely because it prevents intermixing of the $\mathrm{Ni}$ and $\mathrm{Nb}$. Our model junction simulates this geometry, via supercells in the plane normal to the stack to account for the lattice mismatch (see Fig. 1 and Supplementary Note 2), though we do not include the $\mathrm{Cu}$ layers. We anticipate that $\mathrm{Cu}$ spacers will mainly affect transmission matrix elements rather than the dependence of the supercurrent on ferromagnet thickness, which is the main focus of this work. Furthermore, as discussed before, the $\mathrm{Cu}$ spacers will suppress the direct interaction between the ferromagnet and the superconductor and reduce the inverse proximity effect justifying Andreev approximation for the boundary conditions, see Eq. (14). Therefore, we consider only the simplified $\mathrm{Nb} / \mathrm{Ni} / \mathrm{Nb}$ stack and vary the number of layers (atomic planes) of $\mathrm{Ni}$. In addition, we also consider the effect of different crystallographic orientations of the $\mathrm{Ni}$ planes and investigate $\mathrm{Nb} / \mathrm{Ni}(110) / \mathrm{Nb}$ junctions in Supplementary Note 3.

\section{Band structure of $\mathrm{Nb} / \mathrm{Ni} / \mathrm{Nb}$ junctions}

To make a $\mathrm{Nb} / \mathrm{Ni}$ superlattice, the unit cells of the $\mathrm{Nb}$ and $\mathrm{Ni}$ regions in the plane normal to the interface must be coincident. This is complicated by the severe lattice constant mismatch, and also the incompatibility of the (110) and (111) atomic planes. It is necessary to construct superlattices with $\mathrm{Nb}(110)$ and $\mathrm{Ni}(111)$ both rotated to the $z$-axis and with lattice vectors in the plane coincident. A supercell with nearly coincident vectors was found (see Supplementary Note 2 for details). By applying a small shear strain to the $\mathrm{Ni}$, the lattice vectors are made exactly coincident. Figure $5 \mathrm{a}$ shows the $\mathrm{Nb}(110)$ surface supercell and the $\mathrm{Ni}(111)$ surface supercell with equal lattice vectors used to match the $\mathrm{Nb}$ / $\mathrm{Ni}$ interfaces. Each atomic plane of $\mathrm{Ni}(111)$ contains 14 atoms and each atomic plane of $\mathrm{Nb}(110)$ consists of 10 atoms. The atomic structure of the $\mathrm{Nb}(110) / \mathrm{Ni}(111) / \mathrm{Nb}(110)$ for 5 layers (atomic planes) of $\mathrm{Ni}$ is shown in Fig. 1c.

Next, we performed self-consistent DFT calculations within the local density approximation (LDA) in order to obtain the relaxed structure and corresponding electronic structure. For the smallest structures, we performed a constrained optimization. Only the atoms in the planes closest to the $\mathrm{Nb} / \mathrm{Ni}$ interfaces are allowed to relax to facilitate the stacking of arbitrarily large cells. The $\mathrm{Nb} / \mathrm{Ni}$ interplanar spacing has also been optimized to minimize the total energy, see Supplementary Methods for more details.
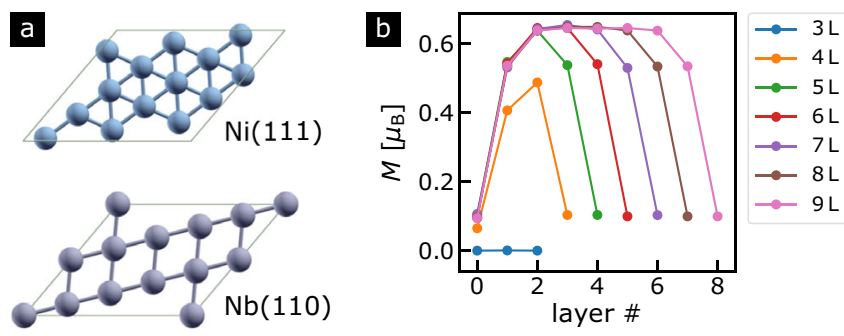

Fig. 5 Magnetization of $\mathbf{N b} / \mathbf{N i} / \mathbf{N b}$ junction. a Top view of the $\mathrm{Nb}$ (110) and $\mathrm{Ni}(111)$ surface supercells used to build the $\mathrm{Nb} / \mathrm{Ni}$ interfaces and the $\mathrm{Nb}(110) / \mathrm{Ni}(111) / \mathrm{Nb}(110)$ stacks shown in Fig. 1c. The surface supercell is defined from two $2 \mathrm{D}$ vectors $\mathbf{a}_{1}=$ $[10.8,0] \AA$ and $\mathbf{a}_{2}=[6.03,7.11] \AA$ with periodic boundary condition in the $2 \mathrm{D}$ plane. The corresponding reciprocal space defines the $2 \mathrm{D}$ $\mathbf{k}_{\|}$vectors used in the calculations. Each atomic plane of $\mathrm{Ni}(\mathrm{Nb})$ contains $14(10)$ atoms of $\mathrm{Ni}(\mathrm{Nb})$. b Magnetic moment profile of $\mathrm{Nb}$ (110)/Ni(111)/ $\mathrm{Nb}(110)$ junctions for different thicknesses of $\mathrm{Ni}$ (from 3 to 9 layers). The value of the moment is an average over the moments of the $14 \mathrm{Ni}$ atoms in each atomic plane. Note the magnetic dead layer at the $\mathrm{Nb} / \mathrm{Ni}$ interfaces and that all moments vanish for the shortest junction made of 3 layers of $\mathrm{Ni}$.

Once the structure is determined, one can determine the normalstate thermodynamic and transport properties of the junction, e.g., calculate the magnetization profile and spin-resolved conductance through the junction as a function of Ni thickness. For transport calculations, we use a layer transport technique ${ }^{47}$ which employs the atomic spheres approximation (ASA). Careful checks were made of ASA band structures of elemental $\mathrm{Nb}$ and $\mathrm{Ni}$, and also superlattices, to confirm that they are very similar to the full potential linear muffin-tin orbital (LMTO) DFT-LDA ones.

We find that the magnetic properties of $\mathrm{Ni}$ are sensitive to their local environment, indicative of the itinerant ferromagnetism. As shown in Fig. 5b, the magnetization profile is non-uniform in the junction with averaged magnetic moments per atom being suppressed near the $\mathrm{Nb}$ interface. For thickness larger than 4 layers, one recovers the bulk value of $\sim 0.6 \mu_{\mathrm{B}}$ in the middle layers, away from the $\mathrm{Nb} / \mathrm{Ni}$ interfaces. The averaged moment drops down towards the edges and becomes considerably reduced down to $\sim 0.1 \mu_{\mathrm{B}}$ at the interface with $\mathrm{Nb}$. The strong reduction of magnetism is exemplified for the short junction with three layers of $\mathrm{Ni}$ where the moments on the $\mathrm{Ni}$ atoms have completely vanished. Such a non-uniform magnetic moment dependence in $\mathrm{Nb} / \mathrm{Ni} / \mathrm{Nb}$ junctions affects superconducting properties of the SFS junctions in a non-trivial way. For example, $\mathrm{Nb} / \mathrm{Ni} / \mathrm{Nb}$ junctions thinner than four layers of $\mathrm{Ni}$ behave as essentially SNS junctions.

It is well-known that the LDA tends to overestimate local moments $M$ in itinerant magnets ${ }^{48}$ because spin fluctuations reduce the average moment ${ }^{49}$, and underestimate $M$ when local moments are very large ${ }^{46}$. For Ni, LDA yields $M$ in good agreement with the experiment, but this is likely an artifact of an accidental cancellation of errors. Most important for transport is the exchange splitting $V_{\text {ex }}$, which the LDA predicts to be $0.6 \mathrm{eV}$, about twice larger than the experimental value of $0.3 \mathrm{eV}^{50}$. It is possible to reproduce both $M$ and $V_{\text {ex }}$ at the same time, but a high-level theory, potentially including spin-orbit coupling, is needed to surmount both kinds of errors inherent in the $\operatorname{LDA}^{46,51}$. The high cost and poor scaling of such a theory are not practical for these junctions, so we elect to stay within the LDA and scale the selfconsistently calculated $V_{\text {ex }}$. This was the approach Karlsson and Aryasetiawan used to calculate the spin-wave spectra in $\mathrm{Ni}^{52}$. Scaling of $V_{\text {ex }}$ can be accomplished using different approaches, e.g., by adding some effective magnetic field to simulate the effect of spin fluctuations. Since $\mathrm{Ni}$ is a simple case with a nearly linear relation between $M$ and $V_{\text {ex }}$, the band structure hardly depends on 
the details in which the LDA potential is modified. Here, we first perform fully self-consistent calculations. Then, to construct the potential for transport properties, we rescale the spin component of the density by a constant factor, which we denote as $M / M_{0}$. This enables parametric studies of transport as a function of $V_{\mathrm{ex}}$. $M / M_{0}=0.5$ yields the observed $V_{\text {ex }}=0.3 \mathrm{eV}$, and we use this scaling unless stated otherwise.

The conductance per unit of area, $G / A$, is shown in Fig. $3 \mathrm{~b}$. It is weakly dependent on the thickness, $w$, of the magnetic layer, as expected for a metallic system in the absence of disorder.

\section{Superconducting transport}

We now focus on superconducting properties. The dependence of the supercurrent on the phase difference $\phi$ for 5,8 , and $11 \mathrm{Ni}$ layers is shown in Fig. 3c-e. One can present current-phase relation, $J(\phi)$ in a form of a Fourier series

$$
J(\phi)=\sum_{n \geqslant 1} J_{n} \sin (n \phi)
$$

In the 0 -junction mode, the first term in the Fourier series dominates with $J_{1}>0$ [see solid black line in Fig. 3c]. In the $\pi$ junction case [Fig. 3e], the supercurrent is also mostly defined by the first harmonic but with $J_{1}<0$. Close to the $0-\pi$ transition $J_{1}$ dies out so that the behavior is governed by higher Fourier harmonics ${ }^{53}$, e.g., for $8 \mathrm{Ni}$ layers supercurrent has mostly second harmonic, $J_{2}$, shown by the dashed black line in Fig. $3 \mathrm{~d}$.

Figure 6 shows the critical current density, $J_{c}=\max |J(\phi)|$, normalized by normal-state conductance, eJ $J_{C} A / G \Delta$, as a function of $w$ (blue squares). Far from the $0-\pi$ transitions, the critical current coincides with the absolute value of the first Fourier harmonic, e $J_{1} \mid A / G \Delta$ (red circles). Since $J_{1}$ contains a sign of the current and has better numerical stability than $J_{c}$, we use this quantity for the analysis. We exclude very thin junctions (three Ni layers or less) from the analysis since the magnetic properties are suppressed there. In particular, for the first data point in Fig. 6 corresponding to three layers of $\mathrm{Ni}$ the magnetization is completely suppressed [see Fig. $5 b$ ] and the ratio $e J_{c} A / G \Delta \approx 2.2$ is significantly higher than the one for thicker $\mathrm{Ni}$ regions with non zero magnetic moments. We compare this value with the result for the short disordered SNS junction. The combination of analytical energy spectrum ${ }^{54}$ with Dorokhov distribution of channel transmissions ${ }^{55,56}$ leads to a ratio

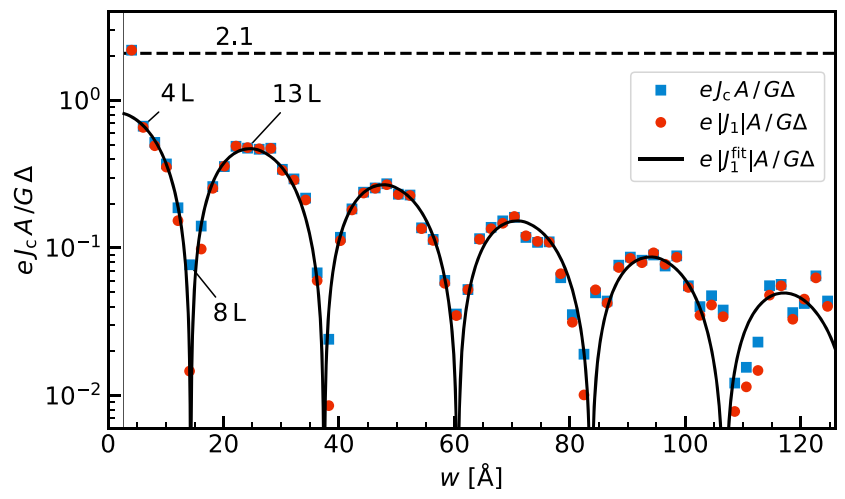

Fig. 6 Dependence of the supercurrent on $\mathrm{Nb}$ thickness. Comparison of critical current density, $J_{c}=\max _{\phi}|J(\phi)|$ (blue squares), the absolute value of first Fourier component for supercurrent, $|J 1|$ [see Eq. (5), red circles], and its fitting $\left|f_{1}^{\text {fit }}\right|$ [Eq. (6), black curve]. All these quantities are "normalized" by normal-state conductance, $G$. of 2.1 (horizontal dashed line in Fig. 6). We attribute this difference to the fact that there is no interfacial disorder in our model.

The $J_{1}$ dependence on $w$ can be fit by the following expression

$J_{1}^{\mathrm{fit}}(w)=\Theta_{\jmath} \exp \left(-w / \xi_{\jmath}\right) \cos \left[\pi\left(w+\delta_{\jmath}\right) / \lambda_{\jmath}\right]$,

where $\Theta_{J}=3.20 \mathrm{~A} / \mu \mathrm{m}^{2}, \xi_{J}=41.1 \AA, \lambda_{J}=23.2 \AA$, and $\delta_{J}=-2.75 \AA$ are fitting parameters. We interpret $\xi_{\text {J }}$ as a decay length, $\lambda_{\text {, as }}$ the "half-period" of the oscillation in $J_{1}$ as a function of $w$, and $\delta_{J}$ as a measure of the suppressed magnetization in Ni layers near the $\mathrm{Ni}$ / $\mathrm{Nb}$ boundaries. $f_{1}^{\text {fit }}(w)$ accurately fits the discrete points $J_{1}$ as shown in Figs. 3a, 6, 7, and 10 by black solid line and black circles, accordingly.

\section{Details of the supercurrent}

In order to gain insight into the evolution of $J_{1}$ with $w$, let us resolve contributions from different $\mathbf{k}_{\|}$. For this, we rewrite Eq. (3) as

$J(\phi)=A \int_{\mathrm{BZ}} \frac{d \mathbf{k}_{\|}}{(2 \pi)^{2}} j\left(\phi, \mathbf{k}_{\|}\right)$,

$j\left(\phi, \mathbf{k}_{\|}\right)=-\frac{e}{\hbar} \frac{1}{A} \sum_{v>0} \frac{\partial \varepsilon_{v}\left(\phi, \mathbf{k}_{\|}\right)}{\partial \phi}$.

Here, $A=76.75 \AA^{2}$ is the area of the surface supercell shown in Fig. 5a. Similar to Eq. (5), we denote the first $\phi$-harmonic of $j\left(\phi, \mathbf{k}_{\|}\right)$ as $j_{1}\left(\mathbf{k}_{\|}\right)$. The evolution of the first Fourier harmonic of the supercurrent $J_{1}$ and $j_{1}\left(\mathbf{k}_{\|}\right)$as a function of $w$ is shown in Fig. 7. Calculations were performed for two different sets of $\mathbf{k}_{\|}$with 290 discrete $\mathbf{k}_{\|}$-points (full black circles) and $4142 \mathbf{k}_{\|}$-points (empty black circles). One can see that both sets give the same result for $J_{1}$, establishing that the $\mathbf{k}_{\|}$integration is well converged. In Fig. 7, "errorbars" denote the standard deviation in $j_{1}\left(\mathbf{k}_{\|}\right)$with respect to the $\mathbf{k}_{\|}$-summed average, $J_{1}(\phi)$. Individual $j_{1}\left(\mathbf{k}_{\|}\right)$is shown by semitransparent horizontal dashes. The important observation is that while $J_{1}$ decays with $w$, the dispersion in $j_{1}\left(\mathbf{k}_{\|}\right)$does not change significantly.

Figure 8 shows color plots of $j_{1}\left(\mathbf{k}_{\|}\right)$corresponding to local extrema of $J_{1}^{\text {fit }}(w)(4,13,25,36,48$, and 60 layers labeled in Fig. 7). For small $w$, one can see that most of all the $\mathbf{k}_{\|}$contributions to $J_{1}$ have the same sign, i.e., positive in the 0 -junction regime and negative in the $\pi$-junction regime. In this regime, the decay is predominantly due to evanescent modes decaying into the junction. For larger $w$, the dephasing mechanism becomes important since the phase offset spread grows with $w$. One can observe the apparition of contributions of the opposite sign for $w \gtrsim 50 \AA$. This dephasing mechanism is mainly due to the variation of the Fermi velocity with $\mathbf{k}_{\|}$, and becomes more important with increasing $w$.

In order to study the distribution of the phase offsets and decay exponents for different modes, we fit the individual $j_{1}\left(\mathbf{k}_{\|}\right)$using an expression analogous to Eq. (6). The set of the resulting fitted curves $f_{1}^{\text {fit }}\left(w, \mathbf{k}_{\|}\right)$for $4142 \mathbf{k}_{\|}$-points are shown by the green semitransparent curves in Fig. 3a. Here, to minimize the numerical "noise," $j_{1}\left(w, \mathbf{k}_{\|}\right)$curves are smoothed over the $2 \mathrm{D} \mathbf{k}_{\|}$space using Gaussian filter with $\sigma_{\mathbf{k}}=0.01 \AA^{-1}$ which is of the order of the Fermi wave vector in $\mathrm{Nb}$. Thus, each data point $j_{1}\left(w, \mathbf{k}_{\|}\right)$ approximately corresponds to a transverse conducting channel. This fitting procedure works reasonably well, e.g., the relationship in Eq. (7a) holds if one replaces $J(\phi)$ by $J_{1}^{\text {fit }}(w)$ and $j\left(\phi, \mathbf{k}_{\|}\right)$by $j_{1}^{\text {fit }}\left(w, \mathbf{k}_{\|}\right)$for $w \gtrsim 10 \AA$. .

The distribution of the fitting parameters for $j_{1}^{\text {fit }}\left(w, \mathbf{k}_{\|}\right)$is shown in Fig. 9. Histograms for decay lengths and half-periods reveal a complicated picture describing different contributions to the supercurrent in real materials. First of all, in Fig. 9a one can see the distribution of the half-periods, $\lambda_{j}$, which is similar to a Gaussian distribution with a mean value $\left\langle\lambda_{j}\right\rangle=23.2 \AA$ and standard deviation $2.8 \AA$. The mean value $\left\langle\lambda_{j}\right\rangle$ is very close to $\lambda_{\text {j }}$ [see text after Eq. (6)] while the spread in $\lambda_{j}$ leads to dephasing and is 


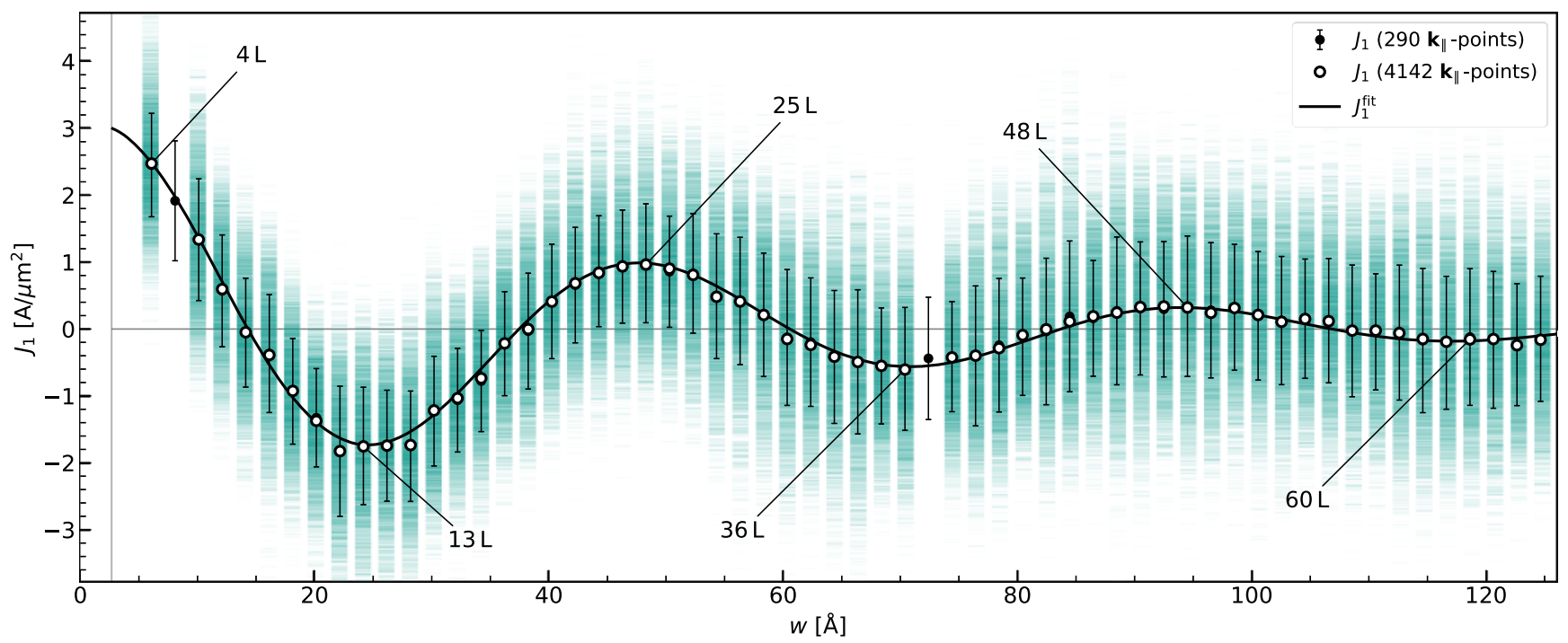

Fig. 7 First Fourier harmonic, $\boldsymbol{J}_{1}$, of the supercurrent as a function of junction thickness, w. Full black circles correspond to $J_{1}$ calculated with $290 \mathbf{k}_{\|}$-points, empty circles (mostly superposed onto the full black circles) correspond to $4142 \mathbf{k}_{\|}$-points. A solid black line is $J_{1}^{\text {fit }}(w)$ [ $J_{1}$ fit given by Eq. (6)]. Green semitransparent dashes show $f_{1}^{\text {fit }}(w)$ contributions [Eq. (7b)] for the individual $\mathbf{k}_{\|}$-points. The vertical "errorbars" correspond to the standard deviation of $j_{1}\left(\mathbf{k}_{\|}\right)$with respect to $J_{1}$. The standard deviation is the same for both sets of $\mathbf{k}_{\|}$-point indicating that these results are independent of the chosen discretization.
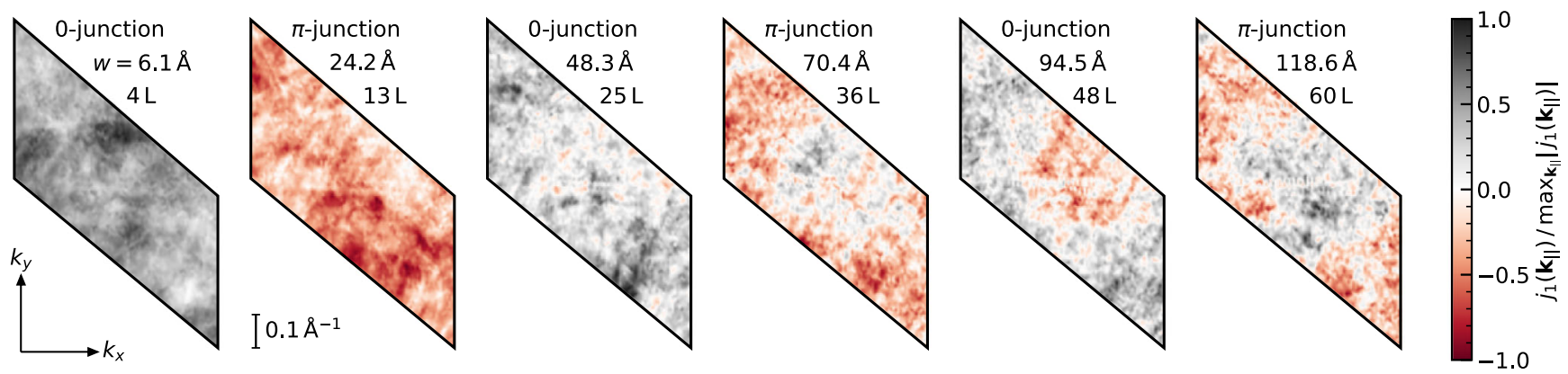

Fig. 8 Colorplot of $\boldsymbol{j}_{\mathbf{1}}\left(\mathrm{k}_{\|}\right)$with $\mathrm{k}_{\|}=\left(\boldsymbol{k}_{\boldsymbol{x}}, \boldsymbol{k}_{\mathbf{y}}\right)$. Each panel corresponds to the local extrema of $J_{1}^{\text {fit }}(w)$ shown in Fig. 7 . For 4 and 13 layers, all the $\mathbf{k}_{\|}$ channels contribute with the same sign. For 48 layers and larger, different $\mathbf{k}_{\|}$channels lose synchronization and contribute to the total supercurrent with different signs.
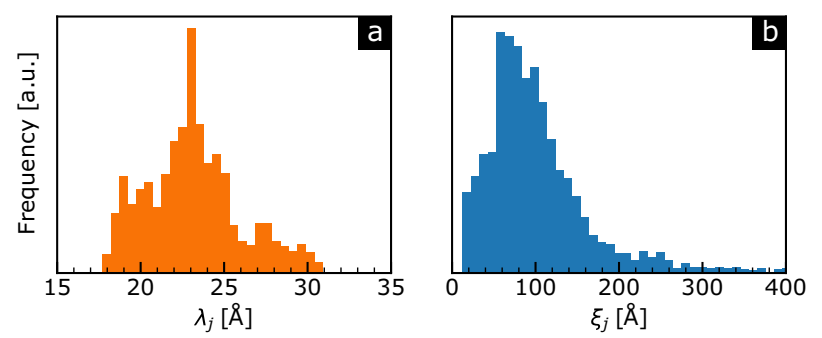

Fig. 9 Fitting parameters for model supercurrent. Analysis of the fitting parameters of $j_{1}^{\text {fit }}(w)$ for individual $\mathbf{k}_{\|}$, shown in Fig. 3a by semitransparent green lines. Distribution of $\mathbf{a}$ half-periods, $\lambda_{j}$, and $\mathbf{b}$ decay lengths, $\xi_{j}$.

responsible for the exponential decay of $J_{1}$ at large $w$. Indeed, it is well-known that the average of an oscillatory function with respect to a random fluctuating phase (described by a Gaussian distribution) results in an exponentially decaying function.

In addition to the dephasing mechanism, the decay of the supercurrent originates from the evanescent modes. The histogram for decay lengths, $\xi_{j}$ is shown in Fig. 9b. Here, small $\xi_{j}$ corresponds to fast-decaying $j_{1}^{\text {fit }}\left(w, \mathbf{k}_{\|}\right)$, large $\xi_{j}$ is responsible for non-decaying modes (i.e., modes with the decay exponents larger than the junction thickness). The right-skewed distribution of the decay exponents has a mean value of $\left\langle\xi_{j}\right\rangle=108 \AA$ which is much larger than $\xi_{j}$ in Eq. (6). The shoulder at small $\xi_{j}$ presumably corresponds to the evanescent mode decay comprising of $d$ bands 2 and 3, see Table 1 , whereas the tail at large $\xi_{j}$ originates predominantly from band 6. Overall, one can see that a fit with a single decay exponent, discussed in Eq. (6), is quite oversimplified for an $\mathrm{Nb} / \mathrm{Ni} / \mathrm{Nb}$ junction considered here.

\section{Exchange splitting}

We now turn to the discussion of the effect of exchange splitting energy on the supercurrent in MJJs. So far we have used $M / M_{0}=$ 0.5 , which yields the experimentally observed $V_{\text {ex }}=0.3 \mathrm{eV}$. It is interesting to investigate how a ferromagnet with a different $V_{\mathrm{ex}}$ (but otherwise the same band structure as Ni) would affect the $w$ dependence of $J_{1}$. In Fig. 10, we show the results for parametric variations in $M / M_{0}$. One can see in Fig. 10a that the half-period, $\lambda_{\jmath}$, and the decay length, $\xi_{\text {, }}$ strongly depend on $M$. Here, black points correspond to $M / M_{0}=0.5$, and the self-consistent calculations with no rescaling correspond to $M / M_{0}=1$. In order to understand how $\lambda_{\jmath}$ and $\xi_{J}$ depend on $M$, we perform the fitting procedure Eq. (6) for different magnetic moments and plot, in Fig. 10b, the 

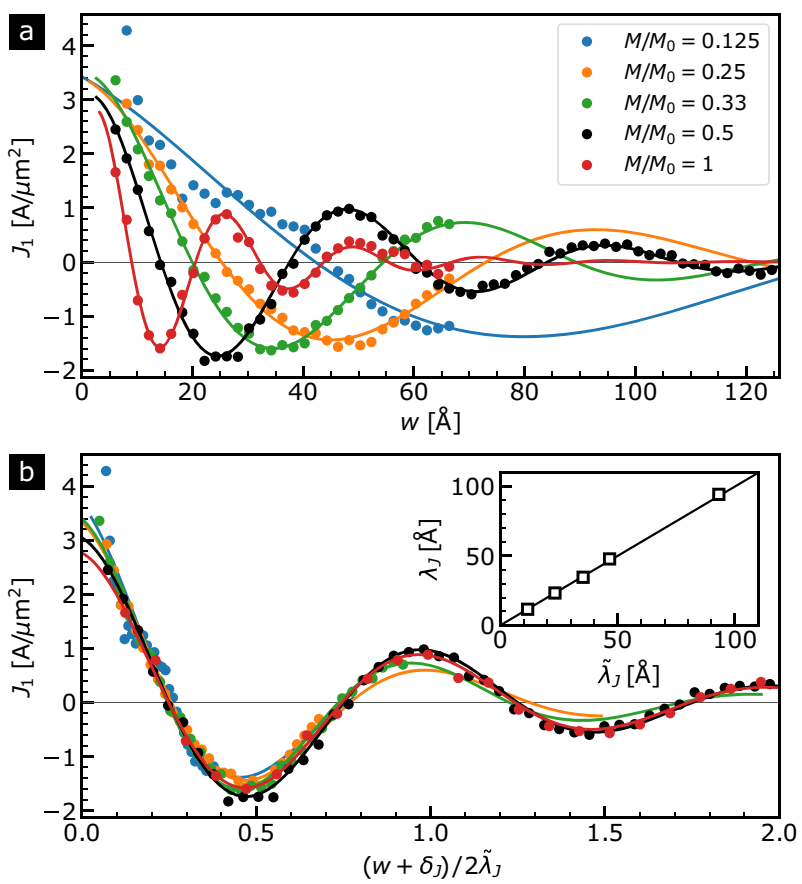

Fig. 10 Magnetic moments rescaling. $J_{1}$ for different rescaling of the magnetic moments $M / M_{0}$ as a function of a the thickness, $w$, and b the rescaled thickness, $\left(w+\delta_{J}\right) / 2 \tilde{\lambda}_{\jmath}$, where $\delta_{\jmath}$ is the fitting parameter in Eq. (6) and $\tilde{\lambda}_{J}=\left(M_{0} / M\right) 11.6 \AA$. $J_{1}$ values are shown by circles; corresponding fittings $J_{1}^{\text {fit }}(w)$ are shown by lines of the same color. (Inset) Half-period, $\lambda_{\text {, }}$ fitted using Eq. (6) vs. $\tilde{\lambda}_{\text {. }}$.

supercurrent density as a function of the rescaled thickness, $(w+$ $\left.\delta_{J}\right) / 2 \tilde{\lambda}_{\text {, with }} \tilde{\lambda}_{J}=\left(M_{0} / M\right) 11.6 \AA$. Remarkably $J_{1}$, as a function of the rescaled thickness, collapses to the same universal curve. The inset demonstrates that the fitted half-period, $\lambda_{ر}$, is proportional to $1 / M$ showing that the oscillation period scales linearly with the inverse of $V_{\mathrm{ex}}$ in this parameter range.

Note that deviations from the linear regimes become significant for $M / M_{0} \gtrsim 1.7$. For the clarity of the data, we do not show the results for $M / M_{0}>1$ in Fig. $10 \mathrm{~b}$.

\section{Different orientations of Ni plane}

We now discuss the difference in crystal orientation in $\mathrm{Nb} / \mathrm{Ni} / \mathrm{Nb}$ junctions. We have performed calculations for $\mathrm{Nb} / \mathrm{Ni} / \mathrm{Nb}$ junctions built from stacking the $\mathrm{Ni}$ atomic planes in the (110) orientation instead of the (111) orientation. The results are given in Supplementary Note 3. Qualitatively, the same physics holds for both stacks built from (111) and (110) Ni planes. However, our calculations show that the actual value for the period of oscillation and for the current decay depend crucially on the details of the electronic structure of the junctions, such as the relative crystal orientation.

\section{Spin-orbit coupling}

Finally, we considered the effect of spin-orbit coupling in SFS junctions. Spin-orbit coupling leads to the mixing of the minority and majority channels and may change current-phase relationship. The interplay between Zeeman splitting and spin-orbit coupling have been discussed in ref. ${ }^{45}$; the regime of interest is the Zeemanfield-dominated regime considered there. Indeed, we find that SOC in $\mathrm{Ni}$ is much smaller than the exchange splitting because of the low atomic number of Ni. As we show in Supplementary Note 4, the SOC in Ni-based MJJ considered here does not change the qualitative picture described above but rather leads to small quantitative changes to the Josephson current.

\section{DISCUSSION}

In this paper, we identified two generic mechanisms for the decay of the supercurrent with junction thickness: (i) exchangesplitting induced gap opening for minority or majority carriers and (ii) dephasing between different modes due to the significant quasiparticle velocity dispersion with the transverse momentum. It was previously believed that disorder in the ferromagnet is mainly responsible for the supercurrent decay in SFS junctions. In the present work, we have shown that band structure effects also contribute to the critical current suppression and thus provide an upper bound for the supercurrent in an ideal (i.e., disorder-free) structure.

We found that the $\mathrm{Nb} / \mathrm{Ni} / \mathrm{Nb}$ junction is a suitable system for comparison with the simulations because of the long mean free path in $\mathrm{Ni}$ relative to the junction thickness and the quasiballistic nature of quasiparticle propagation in the ferromagnet. We have found good agreement with published experimental data for the half-period of the critical current oscillations: $\lambda_{\jmath} \approx$ $23 \AA$ [see Eq. (6) and text after it] vs. $\approx 26 \AA$ in the experiment, ref. ${ }^{22}$. We have also found that the critical current decays exponentially with the ferromagnet thickness $w$. This is to be contrasted with previously assumed algebraic decay based on results for the clean SFS junctions using a simple parabolic-like band structure. We believe that in measured $\mathrm{Nb} / \mathrm{Ni} / \mathrm{Nb}$ junctions with $w \lesssim 50 \AA$ the mechanism (i) is likely to be responsible for the supercurrent decay. This finding is crucial for the material and geometry optimization of MJJs and superconducting magnetic spin valves.

Understanding the interplay of band structure effects and disorder in MJJs is an interesting open problem. We believe that interfacial disorder due to, for example, the surface roughness will mix different $\mathbf{k}_{\|}$modes and will lead to a larger spread of half-periods. This, in turn, will further enhance the dephasing mechanism (ii) of the supercurrent decay discussed here. Strong disorder in the bulk (i.e., mean free path much smaller than junction thickness $w$ ) would lead to the diffusive motion of quasiparticles in the ferromagnet which is a significant departure from the quasi-ballistic junction limit considered here. We think that bulk disorder would induce even more dephasing between different modes because phase offsets in this case will depend on different random trajectories of minority and majority carriers. We, therefore, believe that bulk disorder will lead to even stronger decay of the supercurrent with junction thickness, $w$.

\section{METHODS}

We develop a numerical method to perform realistic simulations of MJJs using a combination of first-principles DFT and BdG calculations. The former is used to obtain the normal-state properties (e.g., band structure, Fermi velocities, and magnetization) and to calculate the normal scattering matrices through the inhomogeneous 3D realistic junctions. As a next step, we take superconductivity into account and calculate supercurrent through the stack assuming the short junction limit.

\section{Normal transport: ab initio description}

To calculate the normal scattering matrix we use the Questaal package for electronic structure calculations based on the LMTO method ${ }^{57}$. It calculates the full non-linear, i.e., nonequilibrium, transport properties of an infinite system describing a central (C) region cladded by two semi-infinite left $(\mathrm{L})$ and right ( $\mathrm{R})$ leads ${ }^{47,58}$, as represented below

$$
\overbrace{\ldots|\mathcal{L}| \mathcal{L}}^{\mathrm{L}}|\overbrace{\mathrm{PL}_{0}\left|\mathrm{PL}_{1}\right| \ldots \mid \mathrm{PL}_{L-1}}^{\mathrm{C}}| \overbrace{\mathcal{R}|\mathcal{R}| \ldots}^{\mathrm{R}}
$$

The LCR system [Fig. 1c] can be partitioned into an infinite stack of principal layers (PLS) which interact only with their nearest neighbors. This is possible because the screened LMTO structure constants are 
short-ranged ${ }^{59}$. In the present case, the $C$ region consists of the ferromagnet, plus two layers of $\mathrm{Nb}$ at the LC and CR interfaces respectively. This is the range over which the perturbation from $C$ significantly modifies the potential in the $\mathrm{L}$ or $\mathrm{R}$ region. To construct the $\mathrm{Nb} / \mathrm{Ni} / \mathrm{Nb}$ stack, coincident site lattices for $\mathrm{Ni}$ and $\mathrm{Nb}$ must be found (details of how this was accomplished are given in Supplementary Note 2). Planes of coincident site lattices are stacked to form the $\mathrm{Nb} / \mathrm{Ni} / \mathrm{Nb}$ structures. Figure 5a shows the $\mathrm{Nb}$ and Ni planes we used, which are denoted here as "surface supercells."

The electronic current flows along the $z$-direction, perpendicular to the PLs lying in the $x y$-plane (transverse direction), see Fig. 1c. Periodic boundary conditions are used within each PL. The corresponding reciprocal space defines the two-dimensional (2D) $\mathbf{k}_{\|}$vectors, i.e., the transverse modes, used in the calculations. The $\mathbf{k}_{\|}$mesh is discretized and integrals over $\mathbf{k}_{\|}$are performed numerically.

The electronic structure of the $C$ region can be separated from $L$ and $R$ regions through self-energies, $\Sigma_{L}$ and $\Sigma_{R}$, that modify the Hamiltonian of the $C$ region. They are most easily calculated if the potential of each $\mathrm{PL}$ in the $\mathrm{L}$ or $\mathrm{R}$ region is identical all through the bulk region. This is the reason for adding a few $\mathrm{Nb}$ layers folded into the $\mathrm{C}$ region. Thus the periodically repeating unit cells in the $L$ and $R$ regions can be safely assumed to have the potential of the bulk crystal. To construct the selfenergies, the potentials of the PL in an infinite stack are needed. These potentials are functions only of the $\mathrm{PL}$ in their own region and may be calculated in several ways. $\Sigma_{L}$ and $\Sigma_{R}$ are obtained from "surface" Green's function (a fictitious system that consists of a semi-infinite stack of $\mathrm{PL}$, each with the same potential). Note that the potential of the $\mathrm{C}$ region is calculated self-consistently. This is important, as the local moments of $\mathrm{Ni}$ are small at the boundary layers, and build up gradually, see Fig. $5 \mathrm{~b}$.

With the potential in hand, the normal-state transport can be calculated using scattering formalism ${ }^{58,60}$. For this, knowing the retarded Green's function, $\mathcal{G}^{r}$, of the junction is sufficient. However, this is not the case for the Josephson current: the individual eigenfunctions are required. Within a Green's function framework, $\mathcal{G}^{r}$ must be organized by normal modes which correspond to the eigenstates of the $L$ and $R$ leads for a prescribed energy $E$. In the PL representation, the Hamiltonian has been discretized into the linear combinations of the LMTO basis functions, and the normal modes are represented as eigenvectors of these basis functions. The Schrödinger equation becomes a differential equation in the PL basis functions ${ }^{61}$. Eigenvectors are calculated by solving a quadratic eigenvalue problem ${ }^{61,62}$, whose eigenvalues correspond to $\exp \left( \pm i k_{z, n} a\right)$, where $a$ is the thickness of the PL. The wavenumber $k_{z, n}$ of the normal mode $n$ can be complex, but to correspond to a propagating mode $k_{z, n}$ must be real. By solving the equation as a function of the energy $E$, one gets all the eigenvalues and eigenvectors which provide the information needed to construct the self-energies $\Sigma_{L}$ and $\Sigma_{R}$ (for each $\mathbf{k}_{\|}$and each spin $\sigma$ ). Note that, in the mode basis, the imaginary part of the self-energies is proportional to the (band) velocity of the modes, and is diagonal for nondegenerate modes ${ }^{62,63}$.

The retarded Green's function $\mathcal{G}^{r}$ of the $C$ region (connected to the $L$ and $\mathrm{R}$ leads) can be written as a matrix in the normal mode basis,

$$
\begin{aligned}
\mathcal{G}_{\sigma}^{r}\left(E, \mathbf{k}_{\|}\right)= & \left\{\left[\mathcal{G}_{\mathrm{C}, \sigma}^{r}\left(E, \mathbf{k}_{\|}\right)\right]^{-1}\right. \\
& \left.-\Sigma_{\mathrm{L}, \sigma}\left(E, \mathbf{k}_{\|}\right)-\Sigma_{\mathrm{R}, \sigma}\left(E, \mathbf{k}_{\|}\right)\right\}^{-1},
\end{aligned}
$$

where $\mathcal{G}_{C}^{r}$ is the Green's function of the isolated $C$ region. On this basis, $\mathcal{G}^{r}$ is decomposed into four blocks

$$
\mathcal{G}_{\sigma}^{\mathrm{r}}\left(E, \mathbf{k}_{\|}\right)=\left[\begin{array}{ll}
\mathcal{G}_{\mathrm{LL}, \sigma}^{\mathrm{r}}\left(E, \mathbf{k}_{\|}\right) & \mathcal{G}_{\mathrm{LR}, \sigma}^{\mathrm{r}}\left(E, \mathbf{k}_{\|}\right) \\
\mathcal{G}_{\mathrm{RL}, \sigma}^{\mathrm{r}}\left(E, \mathbf{k}_{\|}\right) & \mathcal{G}_{\mathrm{RR}, \sigma}^{\mathrm{r}}\left(E, \mathbf{k}_{\|}\right)
\end{array}\right],
$$

upon projecting onto the propagating modes of the $L$ and $R$ leads. These four quantities and the mode velocities completely determine the normal state transport properties of the junctions.

The transmission matrices are defined by the off-diagonal parts of Eq. (9). More specifically, the transmission coefficients $\left[t_{\mathrm{LR}, \sigma}\right]_{n m}$, connecting $L$ and $R$ regions, are given by ${ }^{60}$

$$
\begin{aligned}
{\left[t_{\mathrm{LR}, \sigma}\right]_{n m}\left(E, \mathbf{k}_{\|}\right)=} & i \sqrt{\left|\left[v_{\mathrm{L}, \sigma}\right]_{n}\left(E, \mathbf{k}_{\|}\right)\right|} \\
& \times\left[\mathcal{G}_{\mathrm{LR}, \sigma}^{\mathrm{r}}\right]_{n m}\left(E, \mathbf{k}_{\|}\right) \sqrt{\left|\left[v_{\mathrm{R}, \sigma}\right]_{m}\left(E, \mathbf{k}_{\|}\right)\right|},
\end{aligned}
$$

where $\left[v_{L, \sigma}\right]_{n}$ and $\left[v_{R, \sigma}\right]_{m}$ are the velocity matrix elements for propagating modes $n$ and $m$ in the $L$ and $R$ leads, respectively. The transmission matrix $t_{R L}$ can be obtained from Eq. (10) by replacing $L \leftrightarrow R$. The reflection coefficients are given by the diagonal blocks of Eq. (9). For instance, on the $L$ side

$$
\begin{aligned}
{\left[r_{\mathrm{LL}, \sigma}\right]_{n n^{\prime}} } & \left(E, \mathbf{k}_{\|}\right)=i \sqrt{\left|\left[v_{\mathrm{L}, \sigma}\right]_{n}\left(E, \mathbf{k}_{\|}\right)\right|} \\
& \times\left[\mathcal{G}_{\mathrm{LL}, \sigma}^{\mathrm{r}}\right]_{n n^{\prime}}\left(E, \mathbf{k}_{\|}\right) \sqrt{\left|\left[v_{\mathrm{L}, \sigma}\right]_{n^{\prime}}\left(E, \mathbf{k}_{\|}\right)\right|}-\delta_{n n^{\prime}} .
\end{aligned}
$$

The reflection matrix $r_{\mathrm{RR}}$ on the $\mathrm{R}$ side is obtained from Eq. (11) by replacing $L \leftrightarrow R$.

We define the normal scattering matrix as

$S=\left[\begin{array}{cccc}r_{\mathrm{LL}, \uparrow} & 0 & t_{\mathrm{LR}, \uparrow} & 0 \\ 0 & r_{\mathrm{LL}, \downarrow} & 0 & t_{\mathrm{LR}, \downarrow} \\ t_{\mathrm{RL}, \uparrow} & 0 & r_{\mathrm{RR}, \uparrow} & 0 \\ 0 & t_{\mathrm{RL}, \downarrow} & 0 & r_{\mathrm{RR}, \downarrow}\end{array}\right]$,

where we omit $E$ - and $\mathbf{k}_{\|}$-dependence for brevity.

The linear-response normal conductance, $G_{\sigma}$, per spin is given by

$\frac{G_{\sigma}}{A}=\frac{e^{2}}{h} \int_{\mathrm{BZ}} \frac{d \mathbf{k}_{\|}}{(2 \pi)^{2}} \sum_{n, m}\left|\left[t_{\mathrm{LR}, \sigma}\right]_{n m}\left(E_{\mathrm{F}}, \mathbf{k}_{\|}\right)\right|^{2}$.

It is calculated at the Fermi energy, $E=E_{\mathrm{F}}$. The total conductance is given by $G=G_{\uparrow}+G_{\downarrow}$. Figure 3 b shows that $G, G_{\uparrow}$, and $G_{\downarrow}$ are almost independent of the junction thickness, $w$.

\section{Superconducting transport: a scattering matrix approach}

Equation (12) is the normal-state scattering matrix for metal-ferromagnet-metal (NFN) structure taking into account reflection at both NF interfaces. To account for superconductivity, we introduce a step-like superconducting pairing potential $\Delta=3.1 \mathrm{meV}$ and use the Andreev approximation to account for electron-hole scattering processes $^{3,9}$. This approach combines the details of the atomic structure of $\mathrm{Nb} / \mathrm{Ni} / \mathrm{Nb}$ and superconductivity within the mean-field approximation.

The direct contact between $\mathrm{S}$ and $\mathrm{F}$ layers, as shown in Fig. $1 \mathrm{a}$, would lead to an interaction between them. The back-action of the ferromagnet on the superconductor (i.e., the inverse proximity effect) results in a spatial dependence of the pairing potential near the SF interfaces ${ }^{64-66}$. In the case of a clean SFS junction model, the self-consistent BdG calculations have been discussed in refs. ${ }^{67-71}$. In typical experimental systems the superconductor is disordered, so the disorder effect on pairing potential needs to be considered, see, e.g., ref. ${ }^{72}$. Furthermore, in recent experiments $\mathrm{S}$ and $\mathrm{F}$ layers are separated by an intermediate spacer layer, which significantly reduces this inverse proximity effect. Thus, to understand the inverse proximity effect in realistic SFS devices one would need to include both of the above mentioned ingredients in the model as well as to take into account the inhomogeneous magnetization in the ferromagnet (see e.g. Fig. 5b) which is outside the scope of this paper. For the sake of clarity, we focus here on a realistic band structure in the ferromagnet and its effect on the supercurrent in the SFS structures. Henceforth, we also neglect the orbital effects of the fringe magnetic field, created by the ferromagnet.

Since $\Delta \ll E_{\mathrm{F}}$, spin-resolved Andreev reflection at $\mathrm{SN}_{\mathrm{L}}$ and $\mathrm{N}_{\mathrm{R}} \mathrm{S}$ interfaces is described by

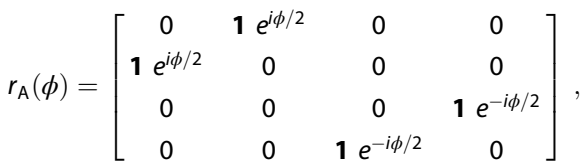

where $\phi$ is the phase difference between left and right superconducting leads and $\mathbf{1}$ is the identity matrix. In the short junction limit, the main contribution to the supercurrent comes from Andreev bound states localized in the junction having energy $\varepsilon_{v}$. The energy spectrum of Andreev states can be obtained using the following equation ${ }^{54}$,

$$
a(\varepsilon)\left[\begin{array}{cc}
0 & r_{\mathrm{A}}^{*}(\phi) \\
r_{\mathrm{A}}(\phi) & 0
\end{array}\right]\left[\begin{array}{cc}
S\left(E_{\mathrm{F}}+\varepsilon, \mathbf{k}_{\|}\right) & 0 \\
0 & S^{*}\left(E_{\mathrm{F}}-\varepsilon, \mathbf{k}_{\|}\right)
\end{array}\right] \psi^{\text {in }}=\psi_{\text {in }},
$$

where $a(\varepsilon)=\sqrt{1-\varepsilon^{2} / \Delta^{2}}+i \varepsilon / \Delta$. The vector $\psi^{\text {in }}=\left[\psi_{\mathrm{e} \uparrow}^{\mathrm{L} \rightarrow}, \psi_{\mathrm{e} \downarrow}^{\mathrm{L}} \rightarrow \psi_{\mathrm{e} \uparrow}^{R \leftarrow}, \psi_{\mathrm{e} \downarrow}^{\mathrm{R} \leftarrow}\right.$, $\left.\psi_{\mathrm{h} \uparrow}^{\llcorner\overrightarrow{ }}, \psi_{\mathrm{h} \downarrow}^{\llcorner\overrightarrow{ }}, \psi_{\mathrm{h} \uparrow}^{R \leftarrow}, \psi_{\mathrm{h} \downarrow}^{R \leftarrow}\right]^{\top}$ corresponds to the electron- and hole-like (e/h) waves in $N_{L}$ and $N_{R}$ regions incident on the $F$ region from the left $(\rightarrow)$ and from the right $(\leftarrow)$.

Simulations show that $S$ is weakly-dependent on $E$ in the range $\left[E_{\mathrm{F}}-\Delta\right.$, $\left.E_{\mathrm{F}}+\Delta\right]$. Therefore, we expand $S\left(E, \mathbf{k}_{\|}\right)$in $E-E_{\mathrm{F}}$ and keep only the leading 
term, i.e., $S\left(E, \mathbf{k}_{\|}\right) \approx S\left(E_{\mathrm{F}}, \mathbf{k}_{\|}\right)=S\left(\mathbf{k}_{\|}\right)$. Using this approximation, one can simplify the quantization condition (15) and reduce it to the matrix eigenvalue problem (see details in ref. ${ }^{73}$ ). This approach allows one to reliably calculate the Andreev bound states spectrum, $\varepsilon_{v}\left(\phi, \mathbf{k}_{\|}\right)$. The zerotemperature supercurrent, $J$, through the junction is given by Eq. 3 . Figure $3 c-e$ shows $J$ as a function of a phase difference, $\phi$. Figure $3 a$ shows the first Fourier harmonic of the supercurrent as a function of the junction thickness, $w$.

\section{DATA AVAILABILITY}

Source data for figures are available from the corresponding author upon reasonable request.

\section{CODE AVAILABILITY}

Links to specific Questaal tutorials are given in Supplementary Methods.

Received: 3 June 2021; Accepted: 22 December 2021; Published online: 28 January 2022

\section{REFERENCES}

1. Josephson, B. Possible new effects in superconductive tunnelling. Phys. Lett. 1, 251-253 (1962)

2. Warburton, P. A. The Josephson effect: 50 years of science and technology. Phys. Educ. 46, 669-675 (2011).

3. Beenakker, C. W. J. Three "universal" mesoscopic Josephson effects. in (eds Fukuyama, H. \& Ando, T.) Transport Phenomena in Mesoscopic Systems, vol. 109, 235-253 (Springer, Berlin, Heidelberg, 1992).

4. Golubov, A. A., Kupriyanov, M. Y. \& Il'ichev, E. The current-phase relation in Josephson junctions. Rev. Mod. Phys. 76, 411-469 (2004).

5. Bardeen, J., Cooper, L. N. \& Schrieffer, J. R. Microscopic theory of superconductivity. Phys. Rev. 106, 162-164 (1957).

6. Buzdin, A. I. Proximity effects in superconductor-ferromagnet heterostructures. Rev. Mod. Phys. 77, 935-976 (2005).

7. Bergeret, F. S., Volkov, A. F. \& Efetov, K. B. Odd triplet superconductivity and related phenomena in superconductor-ferromagnet structures. Rev. Mod. Phys. 77, 1321-1373 (2005).

8. Blamire, M. G. \& Robinson, J. W. A. The interface between superconductivity and magnetism: understanding and device prospects. J. Condens. Matter Phys. 26, 453201 (2014).

9. Eschrig, M. Spin-polarized supercurrents for spintronics: a review of current progress. Rep. Prog. Phys. 78, 104501 (2015).

10. Fulde, P. \& Ferrell, R. A. Superconductivity in a strong spin-exchange field. Phys. Rev. 135, A550-A563 (1964)

11. Larkin, A. I. \& Ovchinnikov, Y. N. Inhomogeneous state of superconductors. Sov. Phys. JETP 20, 762-769 (1965).

12. Buzdin, A. L., Bulaevskil, L. N. \& Panyukov, S. V. Critical-current oscillations as a function of the exchange field and thickness of the ferromagnetic metal (F) in an S-F-S Josephson junction. JETP Lett. 35, 178 (1982).

13. Ryazanov, V. V. et al. Coupling of two superconductors through a ferromagnet: Evidence for a $\pi$ junction. Phys. Rev. Lett. 86, 2427-2430 (2001).

14. Bell, C. et al. Controllable Josephson current through a pseudospin-valve structure. Appl. Phys. Lett. 84, 1153-1155 (2004).

15. Gingrich, E. C. et al. Controllable $0-\pi$ Josephson junctions containing a ferromagnetic spin valve. Nat. Phys. 12, 564-567 (2016).

16. Dayton, I. M. et al. Experimental demonstration of a Josephson magnetic memory cell with a programmable $\pi$-junction. IEEE Magn. Lett. 9, 1-5 (2018).

17. Ryazanov, V. V., Oboznov, V. A., Veretennikov, A. V. \& Rusanov, A. Y. Intrinsically frustrated superconducting array of superconductor-ferromagnetsuperconductor $\pi$ junctions. Phys. Rev. B 65, 020501 (2001).

18. Kontos, T. et al. Josephson junction through a thin ferromagnetic layer: negative coupling. Phys. Rev. Lett. 89, 137007 (2002).

19. Sellier, H., Baraduc, C., Lefloch, Fmc \& Calemczuk, R. Temperature-induced crossover between 0 and $\pi$ states in S/F/S junctions. Phys. Rev. B 68, 054531 (2003).

20. Robinson, J. W. A., Piano, S., Burnell, G., Bell, C. \& Blamire, M. G. Critical current oscillations in strong ferromagnetic $\pi$ junctions. Phys. Rev. Lett. 97, 177003 (2006).

21. Khaire, T. S., Pratt, W. P. \& Birge, N. O. Critical current behavior in Josephson junctions with the weak ferromagnet PdNi. Phys. Rev. B 79, 094523 (2009).
22. Baek, B., Schneider, M. L., Pufall, M. R. \& Rippard, W. H. Phase offsets in the criticalcurrent oscillations of Josephson junctions based on $\mathrm{Ni}$ and $\mathrm{Ni}-\left(\mathrm{Ni}_{81} \mathrm{Fe}_{19}\right)_{x} \mathrm{Nb}_{y}$ barriers. Phys. Rev. Appl. 7, 064013 (2017).

23. Baek, B., Schneider, M. L., Pufall, M. R. \& Rippard, W. H. Anomalous supercurrent modulation in Josephson junctions with Ni-based barriers. IEEE Trans. Appl. Supercond. 28, 1-5 (2018).

24. Aguilar, V. et al. Spin-polarized triplet supercurrent in Josephson junctions with perpendicular ferromagnetic layers. Phys. Rev. B 102, 024518 (2020).

25. Mishra, S. S., Loloee, R. \& Birge, N. O. Supercurrent transmission through Ni/Ru/Ni synthetic antiferromagnets. Appl. Phys. Lett. 119, 172603 (2021).

26. McMillan, W. L. Theory of superconductor-normal-metal interfaces. Phys. Rev. 175, 559-568 (1968)

27. Wolfram, T. Tomasch oscillations in the density of states of superconducting films. Phys. Rev. 170, 481-490 (1968)

28. Kulik, I. O. Macroscopic quantization and the proximity effect in S-N-S junctions. J. Exp. Theor. Phys. 30, 944 (1970).

29. Demers, J. \& Griffin, A. Scattering and tunneling of electronic excitations in the intermediate state of superconductors. Can. J. Phys. 49, 285-295 (1971).

30. Griffin, A. \& Demers, J. Tunneling in the normal-metal-insulator-superconductor geometry using the Bogoliubov equations of motion. Phys. Rev. B 4, 2202-2208 (1971).

31. Entin-Wohlman, O. Effect of a barrier at the superconducting-normal metal interface. J. Low. Temp. Phys. 27, 777-786 (1977).

32. Blonder, G. E., Tinkham, M. \& Klapwijk, T. M. Transition from metallic to tunneling regimes in superconducting microconstrictions: excess current, charge imbalance, and supercurrent conversion. Phys. Rev. B 25, 4515-4532 (1982).

33. Furusaki, A. \& Tsukada, M. Dc Josephson effect and Andreev reflection. Solid State Commun. 78, 299-302 (1991).

34. Furusaki, A., Takayanagi, H. \& Tsukada, M. Josephson effect of the superconducting quantum point contact. Phys. Rev. B 45, 10563-10575 (1992).

35. Furusaki, A. DC Josephson effect in dirty SNS junctions: numerical study. Phys. $B$ 203, 214-218 (1994)

36. de Jong, M. J. M. \& Beenakker, C. W. J. Andreev reflection in ferromagnetsuperconductor junctions. Phys. Rev. Lett. 74, 1657-1660 (1995).

37. Tanaka, Y. \& Kashiwaya, S. Theory of Josephson effect in superconductorferromagnetic-insulator-superconductor junction. Phys. C. 274, 357-363 (1997).

38. Žutić, I. \& Valls, O. T. Spin-polarized tunneling in ferromagnet/unconventional superconductor junctions. Phys. Rev. B 60, 6320-6323 (1999).

39. Radović, Z., Lazarides, N. \& Flytzanis, N. Josephson effect in double-barrier superconductor-ferromagnet junctions. Phys. Rev. B 68, 014501 (2003).

40. Cayssol, J. \& Montambaux, G. Incomplete Andreev reflection in a clean superconductor-ferromagnet-superconductor junction. Phys. Rev. B 71, 012507 (2005).

41. Konschelle, F., Cayssol, J. \& Buzdin, A. I. Nonsinusoidal current-phase relation in strongly ferromagnetic and moderately disordered sfs junctions. Phys. Rev. B 78, 134505 (2008)

42. Tzortzakakis, A. F. \& Flytzanis, N. Josephson Junctions With Spin-Orbit and Spin-flip Interactions. Master's thesis. (University of Crete, 2019). https://arxiv.org/abs/ 1909.10617.

43. Demler, E. A., Arnold, G. B. \& Beasley, M. R. Superconducting proximity effects in magnetic metals. Phys. Rev. B 55, 15174-15182 (1997).

44. Gall, D. Electron mean free path in elemental metals. J. Appl. Phys. 119, 085101 (2016).

45. Cheng, M. \& Lutchyn, R. M. Josephson current through a superconductor/semiconductor-nanowire/superconductor junction: effects of strong spin-orbit coupling and Zeeman splitting. Phys. Rev. B 86, 134522 (2012).

46. Sponza, L. et al. Self-energies in itinerant magnets: a focus on Fe and Ni. Phys. Rev. B 95, 041112 (2017)

47. Faleev, S. V., Léonard, F., Stewart, D. A. \& van Schilfgaarde, M. Ab initio tightbinding LMTO method for nonequilibrium electron transport in nanosystems. Phys. Rev. B 71, 195422 (2005).

48. Aguayo, A., Mazin, I. I. \& Singh, D. J. Why $\mathrm{Ni}_{3} \mathrm{Al}$ is an itinerant ferromagnet but $\mathrm{Ni}_{3} \mathrm{Ga}$ is not. Phys. Rev. Lett. 92, 147201 (2004).

49. Moriya, T. Spin Fluctuations in Itinerant Electron Magnetism (Springer-Verlag, Berlin, 1985).

50. Himpsel, F. J., Knapp, J. A. \& Eastman, D. E. Experimental energy-band dispersions and exchange splitting for Ni. Phys. Rev. B 19, 2919-2927 (1979).

51. Bünemann, J., Gebhard, F., Ohm, T., Weiser, S. \& Weber, W. Spin-orbit coupling in ferromagnetic nickel. Phys. Rev. Lett. 101, 236404 (2008).

52. Karlsson, K. \& Aryasetiawan, F. A many-body approach to spin-wave excitations in itinerant magnetic systems. J. Phys. Condens. Matter 12, 7617-7631 (2000).

53. Stoutimore, M. J. A. et al. Second-harmonic current-phase relation in Josephson junctions with ferromagnetic barriers. Phys. Rev. Lett. 121, 177702 (2018).

54. Beenakker, C. W. J. Universal limit of critical-current fluctuations in mesoscopic Josephson junctions. Phys. Rev. Lett. 67, 3836-3839 (1991). 
55. Dorokhov, O. N. On the coexistence of localized and extended electronic states in the metallic phase. Solid State Commun. 51, 381-384 (1984).

56. Mello, P. A., Pereyra, P. \& Kumar, N. Macroscopic approach to multichannel disordered conductors. Ann. Phys. 181, 290-317 (1988).

57. Pashov, D. et al. Questaal: a package of electronic structure methods based on the linear muffin-tin orbital technique. Comput. Phys. Commun. 249, 107065 (2020).

58. Meir, Y. \& Wingreen, N. S. Landauer formula for the current through an interacting electron region. Phys. Rev. Lett. 68, 2512-2515 (1992).

59. Andersen, O. K. \& Jepsen, O. Explicit, first-principles tight-binding theory. Phys. Rev. Lett. 53, 2571-2574 (1984).

60. Fisher, D. S. \& Lee, P. A. Relation between conductivity and transmission matrix. Phys. Rev. B 23, 6851-6854 (1981).

61. Chen, A.-B., Lai-Hsu, Y.-M. \& Chen, W. Difference-equation approach to the electronic structures of surfaces, interfaces, and superlattices. Phys. Rev. B 39, 923-929 (1989).

62. Fujimoto, Y. \& Hirose, K. First-principles treatments of electron transport properties for nanoscale junctions. Phys. Rev. B 67, 195315 (2003).

63. Wimmer, M. Quantum Transport in Nanostructures: From Computational Concepts to Spintronics in Graphene and Magnetic Tunnel Junctions. Ph.D. thesis. (Universität Regensburg, 2008)

64. Halterman, K. \& Valls, O. T. Proximity effects at ferromagnet-superconductor interfaces. Phys. Rev. B 65, 014509 (2001).

65. Halterman, K. \& Valls, O. T. Proximity effects and characteristic lengths in ferromagnet-superconductor structures. Phys. Rev. B 66, 224516 (2002).

66. Csire, G. et al. Relativistic spin-polarized KKR theory for superconducting heterostructures: oscillating order parameter in the Au layer of $\mathrm{Nb} / \mathrm{Au} / \mathrm{Fe}$ trilayers. Phys. Rev. B 97, 024514 (2018).

67. Halterman, K. \& Valls, O. T. Layered ferromagnet-superconductor structures: the $\pi$ state and proximity effects. Phys. Rev. B 69, 014517 (2004).

68. Halterman, K., Barsic, P. H. \& Valls, O. T. Odd triplet pairing in clean superconductor/ferromagnet heterostructures. Phys. Rev. Lett. 99, 127002 (2007).

69. Halterman, K., Valls, O. T. \& Wu, C.-T. Charge and spin currents in ferromagnetic Josephson junctions. Phys. Rev. B 92, 174516 (2015).

70. Halterman, K. \& Alidoust, M. Josephson currents and spin-transfer torques in ballistic SFSFS nanojunctions. Supercond. Sci. Technol. 29, 055007 (2016).

71. Alidoust, M. \& Halterman, K. Supergap and subgap enhanced currents in asymmetric $\mathrm{s}_{1} \mathrm{fs}_{2}$ Josephson junctions. Phys. Rev. B 102, 224504 (2020).

72. Yagovtsev, V. O., Pugach, N. G. \& Eschrig, M. The inverse proximity effect in strong ferromagnet-superconductor structures. Supercond. Sci. Technol. 34, 025003 (2021).

73. van Heck, B., Mi, S. \& Akhmerov, A. R. Single fermion manipulation via superconducting phase differences in multiterminal Josephson junctions. Phys. Rev. $B$ 90, 155450 (2014).

\section{ACKNOWLEDGEMENTS}

H.N is much indebted to Dimitar Pashov for stimulating discussions about developing the Ouestaal package. The authors express their gratitude to Mason Thomas for the organizational help and discussions at the early stages of the project. The authors acknowledge stimulating discussions with Norman Birge, Anna Herr, Tom Ambrose, Nick Rizzo, and Don Miller.
This work is based on support from the U.S. Department of Energy, Office of Science through the Quantum Science Center (QSC), a National Quantum Information Science Research Center. H.N. and M.v.S. acknowledge financial support from Microsoft Station Q via a sponsor agreement between K.C.L. and Microsoft Quantum. In the late stages of this work M.v.S. was supported by the U.S. Department of Energy, Office of Science, Basic Energy Sciences under Award \# FWP ERW7906.

\section{AUTHOR CONTRIBUTIONS}

H.N. and M.v.S. calculated the normal-state scattering matrices of the ferromagnetic part. I.A.S. and A.E.A. calculated the supercurrent. R.M.L. proposed the initial idea of the method used. All authors participated in technical analysis, results interpretation, and paper writing.

\section{COMPETING INTERESTS}

The authors declare no competing interests.

\section{ADDITIONAL INFORMATION}

Supplementary information The online version contains supplementary material available at https://doi.org/10.1038/s41524-021-00694-3.

Correspondence and requests for materials should be addressed to Hervé. Ness, Ivan A. Sadovskyy, Andrey E. Antipov, Mark van Schilfgaarde or Roman M. Lutchyn.

Reprints and permission information is available at http://www.nature.com/ reprints

Publisher's note Springer Nature remains neutral with regard to jurisdictional claims in published maps and institutional affiliations.

\begin{abstract}
Open Access This article is licensed under a Creative Commons Attribution 4.0 International License, which permits use, sharing, adaptation, distribution and reproduction in any medium or format, as long as you give appropriate credit to the original author(s) and the source, provide a link to the Creative Commons license, and indicate if changes were made. The images or other third party material in this article are included in the article's Creative Commons license, unless indicated otherwise in a credit line to the material. If material is not included in the article's Creative Commons license and your intended use is not permitted by statutory regulation or exceeds the permitted use, you will need to obtain permission directly from the copyright holder. To view a copy of this license, visit http://creativecommons. org/licenses/by/4.0/.
\end{abstract}

(c) The Author(s) 2022 